Ambiances

anbiances Environnement sensible, architecture et espace urbain

3 | 2017

Animer l'espace public ? Entre programmation urbaine et activation citoyenne

\title{
De la machine de la fête baroque à la performance urbaine : éphémère éternel
}

From the Baroque Festival Machine to the Urban Performance: Eternal

Ephemeral

\section{Alessandra Cirafici}

\section{OpenEdition}

\section{Journals}

Édition électronique

URL : http://journals.openedition.org/ambiances/991

DOI : 10.4000/ambiances.991

ISSN : 2266-839X

\section{Éditeur :}

Direction Générale des Patrimoines - DAPA - MCC, UMR 1563 - Ambiances Architectures Urbanités (AAU)

Référence électronique

Alessandra Cirafici, «De la machine de la fête baroque à la performance urbaine : éphémère éternel », Ambiances [En ligne], 3 | 2017, mis en ligne le 10 décembre 2017, consulté le 21 avril 2019. URL :

http://journals.openedition.org/ambiances/991; DOI : 10.4000/ambiances.991

Ce document a été généré automatiquement le 21 avril 2019

\section{cc)}

Ambiances is licensed under a Creative Commons Attribution-NonCommercial-NoDerivatives 4.0 International License. 


\title{
De la machine de la fête baroque à la performance urbaine : éphémère éternel
}

\author{
From the Baroque Festival Machine to the Urban Performance: Eternal \\ Ephemeral
}

Alessandra Cirafici

1 Le point de départ de notre article repose sur une conception de la ville comme manière collective de concevoir l'espace urbain. Un espace urbain que l'on définit et évalue ici à travers ses rituels d'usage, ses performances, et cette profonde sensation de surprise, d'émerveillement et de participation que l'éphémère porte avec lui. Dans le cadre de ces réflexions, le concept d'« éphémère » se divise en deux catégories que nous allons essayer ensuite d'expliciter, en nous référant plus particulièrement au cas de la ville de Naples :

1. Entendu comme catégorie de la pensée du projet et comme stratégie d'intervention, l'éphémère, en interprétant de diverses façons le concept du "provisoire ", est protagoniste du développement urbain, accélère les processus d'invention et enclenche des dynamiques de transposition du provisoire en permanent ;

2. Entendu comme catégorie anthropologique, en interprétant de diverses façons l'idée de la «performance urbaine» en tant qu'événement collectif et temporaire, l'éphémère caractérise les mécanismes d'utilisation temporaire de l'espace public et est protagoniste du développement des processus identitaires et d'appartenance aux lieux urbains.

C'est autour de cette conception que nous tenterons de développer quelques considérations. Nous voudrions nous interroger sur la dimension pour ainsi dire historique de l'éphémère dans l'espace public, en essayant de saisir la signification d'une expérience de créativité toute italienne qui s'est déroulée à cheval entre le XVII et le XVIII ${ }^{e}$ siècle, en relation avec le théâtre de la fête baroque. Toutefois, nous voudrions dégager les aspects qui relèvent d'une tendance - qu'on pourrait qualifier d'« éternelle » - à résoudre, plus ou moins consciemment, la question de la mise en scène urbaine en terme de "performance » : quel rôle l'éphémère a-t-il joué et continue-t-il de jouer dans la définition du processus évolutif de la ville? Nous mettrons en évidence le rôle 
spécifique que peut et doit jouer le " concepteur ", au sens le plus large du terme, à savoir celui qui aujourd'hui est censé gouverner le processus d'animation de l'espace public et son rapport avec la ville même.

\section{La fête baroque : nécessité, invention et allégorie}

3 Au cours de la Renaissance se développa en Italie, en même temps que la scénographie théâtrale, le secteur spécifique de la décoration scénique que constituaient les structures festives dont le but était de fêter les événements de la vie de la cour et le triomphe du catholicisme à travers le faste artificiel d'œuvres éphémères : théâtres pour tournois, naumachies, chars allégoriques, arcs de triomphe, machines à feux d'artifice. Si elles étaient destinées à vanter les vertus d'un prince ou d'exalter l'image d'un État, elles semblaient être en réalité le symbole d'un pouvoir instable et inconsistant dans le vide politique d'un pays fragmenté et soumis à la domination étrangère. Durant le XVII ${ }^{e}$ siècle, les constructions éphémères trouvèrent un terrain particulièrement fertile avec la profusion de la poésie baroque, dont l'effet spectaculaire devint la composante majeure. Les mécanismes ingénieux élaborés par Leonardo à Milan, par Pirro Ligorio à Ferrare, par Palladio à Venise, par Brunelleschi, Vasari, Buontalenti à Florence, laissèrent la place à d'extravagantes interventions, parmi lesquelles se distinguent notamment les machines «capricieuses» de Gian Lorenzo Bernini et de son proche collaborateur, Giovan Paolo Shor, qui avec le concours, entre autres, de Domenico et Carlo Fontana, Pietro da Cortona, Andrea Pozzo, Antonio Picchiatti firent de Rome l'une des capitales de la fête baroque. Plus encore que le théâtre, la fête permettait à un large public de se représenter; l'architecte, avec son art de la mise en scène, jouait le rôle de médiateur.

La mise en scène spectaculaire signée par Gian Lorenzo Bernini à Rome en 1661 pour célébrer la naissance du Dauphin de France - fils de Louis XIV et de Marie Thérèse d'Espagne - se donne à voir dans une image spécialement indiquée pour introduire le sujet. A cette occasion, la Place d'Espagne, encore dépourvue du grand escalier de la Trinité-des-Monts du XVIII siècle mais où l'on pouvait déjà admirer la magnifique Fontaine Barcaccia du Bernin, fut littéralement redessinée par une imposante installation contemporaine. Un véritable paysage contenant tous les symboles reconductibles à l'événement (du dauphin aux enseignes royales, aux couronnes, aux éléments naturels...) parmi lesquels beaucoup, qui, mécanisés, s'animaient grâce à d'ingénieux cabestans, poulies, appareils hydriques comme c'est le cas dans les machines de "derrière les coulisses » typiques des décors de théâtre. Le jour même des célébrations, dans le fracas ambiant, un solennel écroulement détruisit trois mois de travail sous le grondement des feux d'artifice tirés à la fin de l'événement (Gigli, 1997, p. 208).

Disserter sur les éléments de l'esthétique du baroque nous amènerait à déborder le cadre de notre réflexion, puisque ce qui nous intéresse ici, c'est avant tout de mettre en relation la naissance et le triomphe de l'éphémère avec la période pendant laquelle la ville de Rome était en train d'être redessinée. Une époque à laquelle le théâtre et d'une manière générale le spectaculaire et le merveilleux étaient au comble de leur expressivité et alimentaient un imaginaire où des structures colossales prenaient la forme d'architectures monumentales et précaires qui modifiaient temporairement la topographie et le paysage de la ville, en offrant un spectacle sans doute à couper le souffle. Au décor d'allégories et faux paysages, à la fois réels et fantastiques, les appareils éphémères ont été le signe peut-être le plus évident de la dramatisation qui a marqué une 
époque, et surtout une société, la société aristocratique qui courait inexorablement vers son déclin. Une théâtralité où l'exubérant appareil commémoratif et esthétique du rituel catholique - très loin de la sobriété que les canons de la contre-réforme avaient imposé jusque-là - se mélangeait avec le goût pour le théâtre, la musique et le bel canto à l'italienne de façon à être non seulement redécouvert, mais également développé et perfectionné pour devenir enfin un fabuleux et puissant instrument de renaissance de l'Église et de la ville de Rome. Dans ces créations confluaient et se fondaient tous les moyens d'expression, y compris la musique et la poésie, et le renouvellement du goût était souvent facilité par la désinvolture que permettait leur caractère provisoire. Voilà un premier point intéressant : la puissance de l'éphémère en tant que catalyseur d'une expérimentation linguistique et formelle indispensable au processus de construction de l'espace urbain et notamment à la naissance de cette extraordinaire invention qu'est la piazza, espace public par excellence.

$6 \mathrm{Au}$ cours de ces années, l'image de Rome ainsi que son réseau routier vont être entièrement rénovés et repensés : au milieu d'un mélange d'éléments sacrés et profanes, temples classiques et obélisques orientaux, surgissent des raccourcis inédits, des décors grands ouverts entre les étroites ruelles médiévales, telles des scènes immenses et somptueuses d'un théâtre imaginaire. Il s'agit en fait du théâtre du baroque romain dont Bernin, Borromini, Pietro da Cortona et bien d'autres furent des représentants.

7 Toutefois, nous ne souhaitons pas nous attarder sur Rome ni même parler de Vienne, Paris ou Munich où le goût baroque a été importé d'Italie, mais de l'impact de cet art magnifique de manipuler le provisoire pour engendrer le merveilleux sur celle qui, à juste titre, a été considérée comme la capitale du goût européen entre la fin du XVII et le début du XVIII ${ }^{\mathrm{e}}$ siècle, à savoir la ville de Naples. 
Illustration 1 : G.L. Bernini, J.P. Schor, Macchina festiva a Roma a Trinità dei Monti per la nascita del Delfino di Francia 1666 (Rome, Macchina festiva à Trinità dei Monti réalisée à l'occasion de la naissance du Dauphin de France 1666)

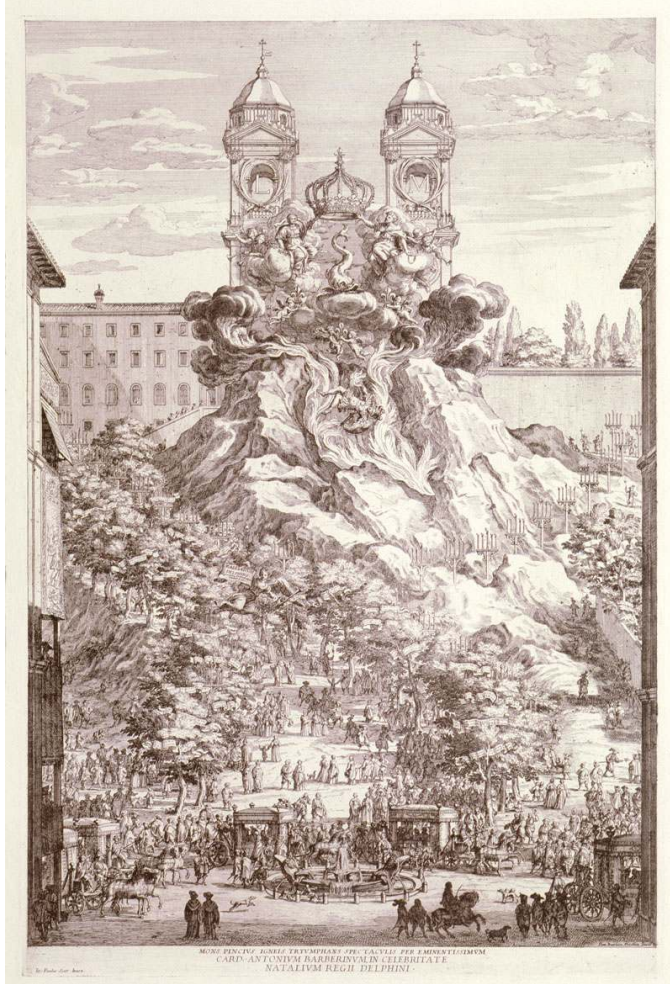

Source et copyright : AA.VV. Capolavori in Festa Effimero Barocco a Largo di Palazzo (gravure). Napoli, 1997, p. 33. Avec l'aimable autorisation d'Ermanno Bellucci (Sovrontendenza Acheologica e curatore del catalogo) 
Illustration 2 : Veduta del Romano Campidoglio con Scalinata che va alla Chiesa d'Aracoeli (Rome, vue du Campidoglio avec les escaliers qui montent vers l'Eglise de l'Aracoeli)

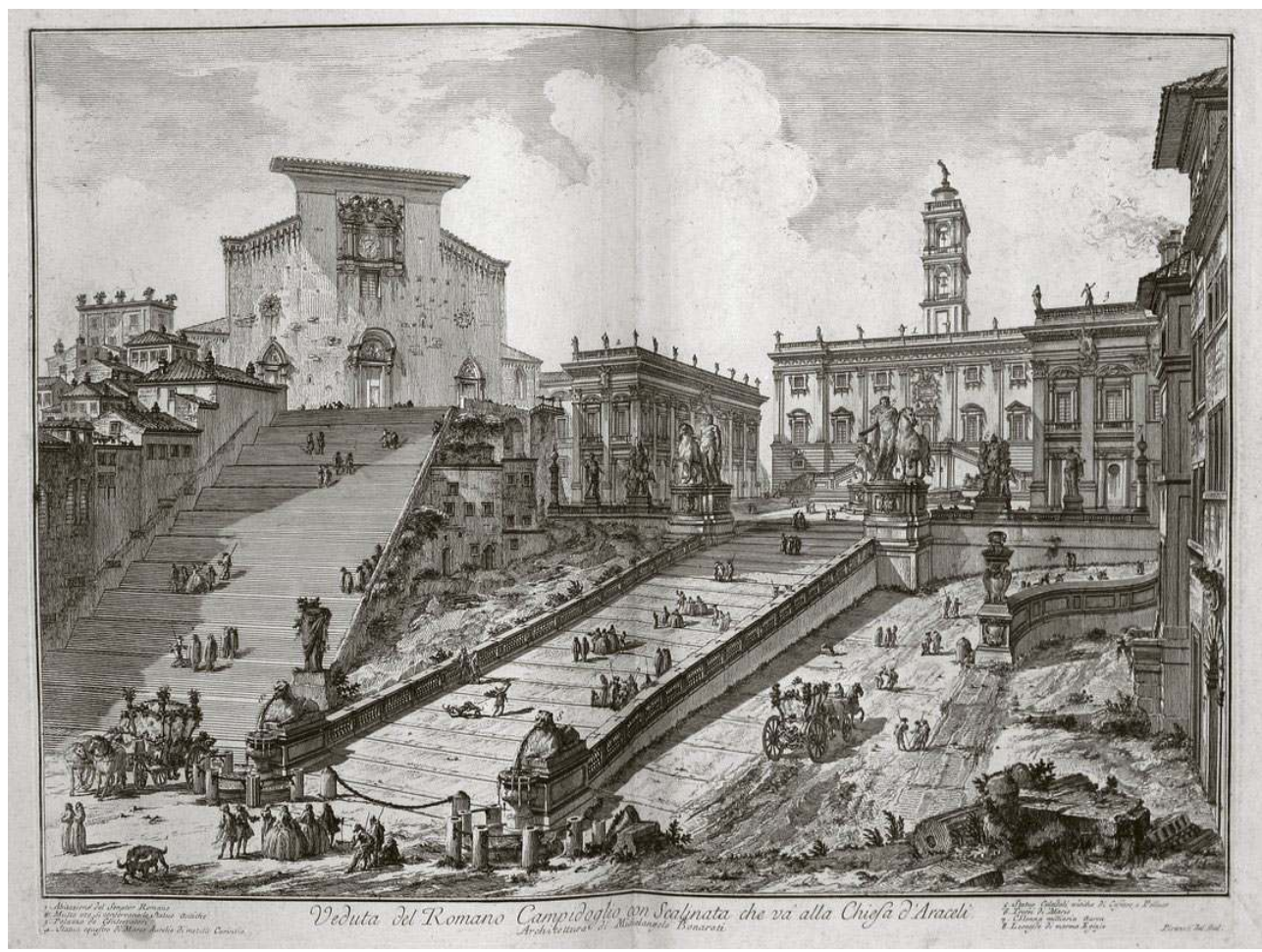

Source et copyright : G.B. Piranesi, Antichità romane, 1756 (gravure). Avec l'aimable autorisation d'Ermanno Bellucci (Sovrontendenza Acheologica e curatore del catalogo).

\section{Naples, éphémère éternel et invention de l'espace public}

Lorsque la culture de l'éphémère arrive à Naples, elle se greffe sur le vécu fort original de la ville. Naples est une ville singulière parce que sa survie a été rythmée par l'équilibre instable entre l'aubaine de la vie et le danger de la destruction. Entre la protection du Saint (San Gennaro, patron de la ville) et la menace du Volcan (le Vésuve). Entre la vie et la mort avec laquelle la ville a noué une relation bien familière, comme le montre le rituel particulier des âmes pezzentelle.

9 L'éphémère est une catégorie constante qui apparaît lors de chaque manifestation de la culture et de la vie à Naples où, au-delà de toute rhétorique, le sentiment du provisoire et du précaire alimenté par un mélange de religiosité, superstition, adaptabilité (aujourd'hui on dirait peut-être « résilience »), s'avère bel et bien tangible.

Dans une page mémorable de son Stadtebilder, Walter Benjamin décrivait la ville de Naples à la fin du XIX ${ }^{e}$ siècle par ces propos :

Poreuse comme cette roche est l'architecture. Édifice et action s'enchevêtrent dans des cours, des arcades et des escaliers. En tout on préserve la marge qui permet à ceux-ci de devenir le théâtre de nouvelles constellations imprévues. On évite le définitif, la marque. Aucune situation n'apparaît telle qu'elle est, prévue pour durer toujours, aucune figure n'affirme "ainsi et pas autrement" C'est ainsi que se développe l'architecture comme une synthèse du rythme communautaire : civilisée, privée et ordonnée... anarchique, imbriquée, rustique. (Benjamin, 2007, p. 6) ${ }^{1}$ 
Illustration 3 : Il Cimitero delle Fontanelle à Naples accueille 40000 squelettes des victimes de la grande peste de 1656 et du choléra de 1836

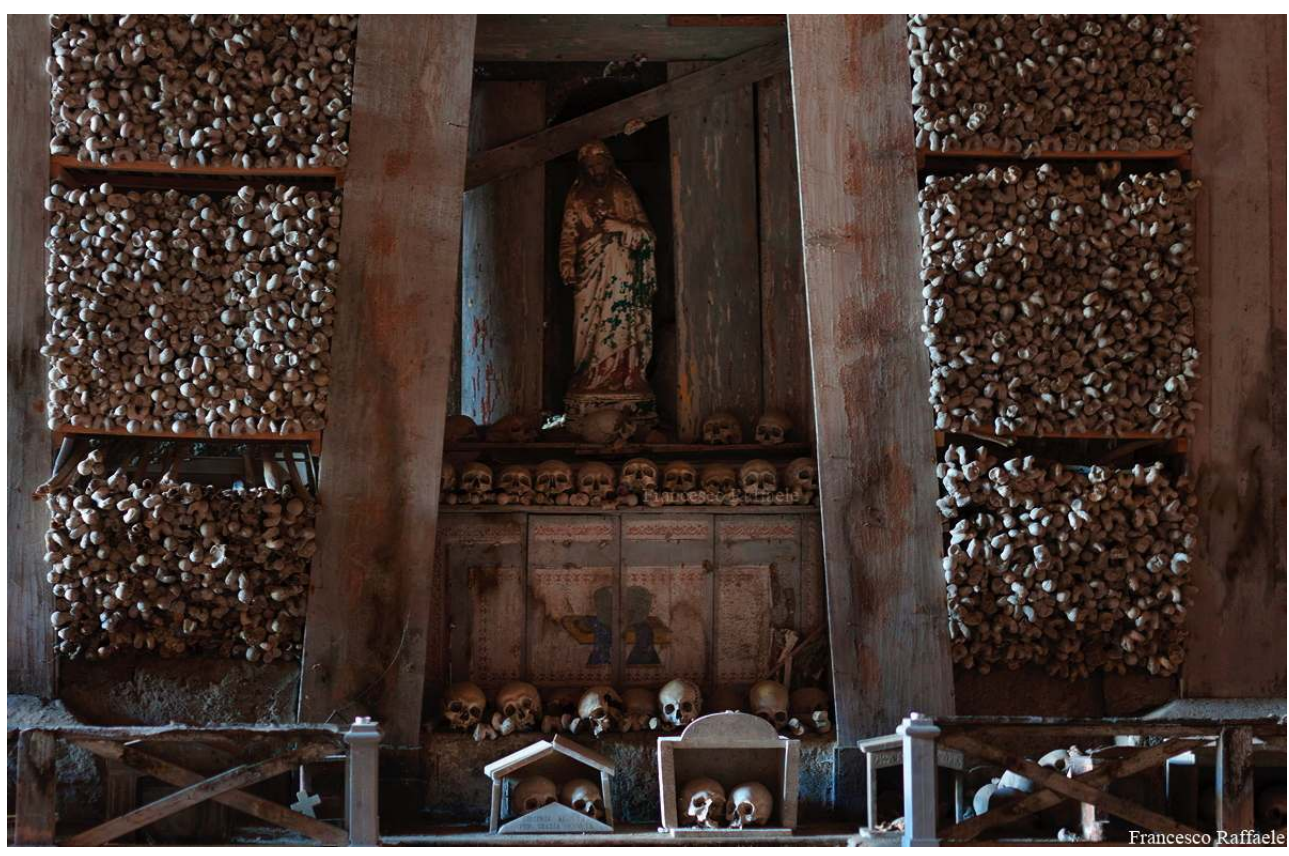

Le cimetière est célèbre grâce à un rituel particulier, nommé « rituel des âmes pezzentelle » (car elles étaient abandonnées). II s'agit donc d'un rituel qui concernait l'adoption et le rangement en ce lieu d'un crâne en échange de protection. A chaque crâne (nommé capuzzella) était liée une âme abandonnée (nommée pezzentella).

Source et copyright: Photo de Francesco Raffaele. Libre de droits.

11 On ne saurait imaginer de description plus lucide et intense et qui demeure incroyablement actuelle. Il apparaît ainsi évident qu'une fois arrivée à Naples, l'énergie $\mathrm{du}$ baroque se greffe sur un terrain très fertile. Elle fait irruption et brise les freins et les règles de l'équilibre de la Renaissance. A la fin du XVII e siècle, Naples se présente comme une ville perméable et déjà prête à s'approprier, exalter et multiplier l'exubérant spectaculaire baroque qui ne la quittera jamais et qui constitue encore aujourd'hui sa connotation authentique. Ici la fonction de propagande liée à l'expérience de l'éphémère se marie avec une tendance innée à la théâtralité et une insolite capacité à apprécier et jouir du moment gai et éphémère de la fête. Ici, une orographie au sédiment très irrégulier qui semble être destinée à devenir un immense théâtre en plein air, réveille l'imagination et rend encore plus spectaculaire l'audace de ses protagonistes (Mancini, 1964).

Si l'on voulait cerner les limites temporelles de l'éclosion de l'éphémère baroque à Naples, l'on pourrait affirmer qu'elle a commencé en 1683 avec l'arrivée en ville de don Gaspar de Haro y Guzmàn, Marquis de Carpio, dans sa qualité de vice-roi et s'est terminée en 1759 avec le départ définitif de Charles de Bourbon pour l'Espagne. Pendant presque un siècle, les Napolitains ne cessèrent de vivre le charme de passionnantes fêtes de cocagne et spectaculaires machines pour la fête. Pour célébrer, par exemple, l'arrivée à Naples de Philippe V d'Espagne (en l'an 1702) la ville fit l'expérience de 48 jours d'affilée de fêtes qui se déroulèrent dans tous ses espaces publics.

13 Bien sûr l'exubérante richesse des appareils scéniques, la redondance, la dispendieuse profusion des mises en scène avaient également une importante fonction politique : la 
célébration du faste d'un prince et de sa cour mais en même temps l'expression métaphorique de sa fragilité et de la caducité de sa gloire. La fête relâche la tension sociale et est vécue par les citadins comme un mécanisme aux rythmes de plus en plus soutenus, comme une sorte d'étourdissement collectif. Naples au fond était et est encore aujourd'hui une ville étourdie et magnifique à la fois, un cas particulier de ville européenne où la mentalité et l'imaginaire collectif ont été fortement influencés par la présence au quotidien du merveilleux et de l'éphémère. Il n'est guère étonnant que, bien après la fin de la période de la fête baroque, quelques-unes des fêtes religieuses (que l'on pense par exemple à celle qui était liée au culte du patron de la ville, San Gennaro ou à celle de la Festadi Piedigrotta, qui était considérée à la fin du XIXe siècle comme la plus connue et la plus ancienne des fêtes en Occident) se soient maintenues dans le temps et qu'encore aujourd'hui on puisse voir des processions, des chars allégoriques et des performances collectives. Il faut également rappeler que la tradition des fuochisti (des artisans qui mettent au point et lancent les feux d'artifice), est encore étroitement liée à la tradition parthénopéenne.

Mais revenons à notre sujet d'étude :

Le Naples baroque est un théâtre festif de rituels, mis en place dans les églises avec de riches chapelles et de précieux autels, dans les palais avec des escaliers majestueux et des portes richement décorées, sur les places avec de nouvelles façades, flèches et fontaines. (Cantone, 1994, p. 245)

Comme on devait faire face à une dimension urbaine réduite et au manque de liquidités des commanditaires, ce qui empêchait un projet complet de rénovation de la ville, comme pour la Rome de Sixte V, les architectes s'appuyaient sur le langage des machines de fête et sur les décorations théâtrales afin d'innover, et de susciter ainsi stupeur et émerveillement à travers l'architecture.

Illustration 4 : Ruiz, Capriccio di festa notturna. Machines pyrotechniques dans le Port de Naples, 1680

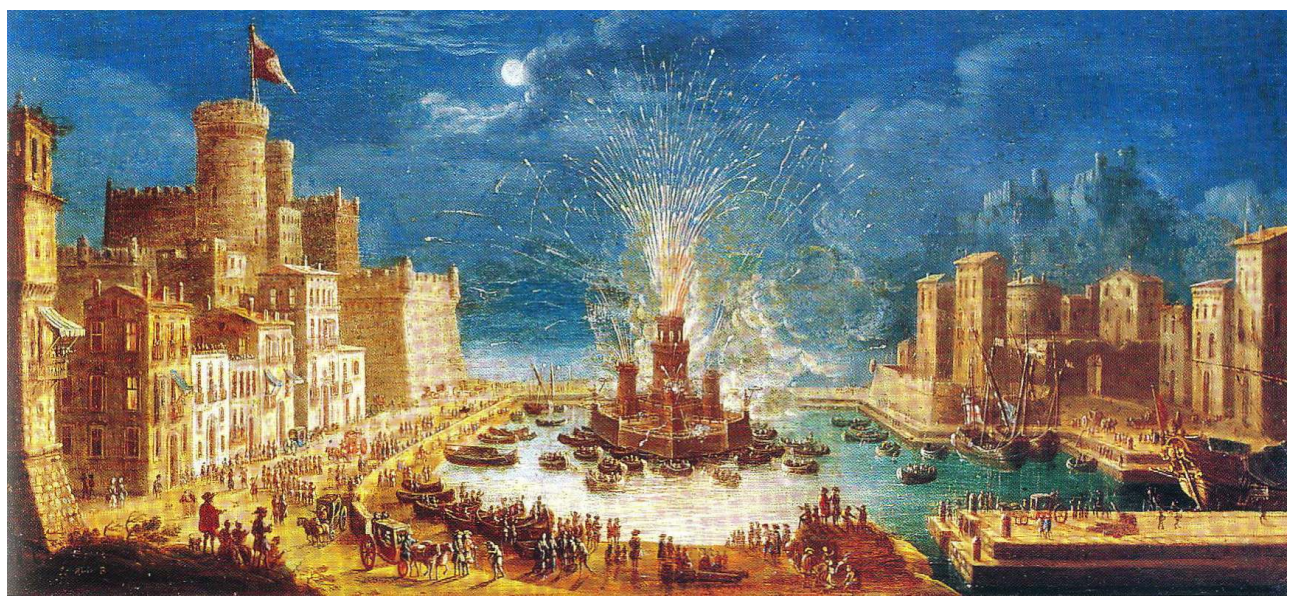

Source et copyright : Huile sur cuivre argenté, collection privée. AA.VV. Capolavori in Festa Effimero Barocco a Largo di Palazzo. Napoli, 1997, p. 171. Avec l'aimable autorisation d'Ermanno Bellucci (Sovrontendenza Acheologica e curatore del catalogo).

Les protagonistes de cette période furent des personnalités telles que Cosimo Fanzago, Ferdinando Sanfelice, Andrea Vaccaro, Niccolò Tagliacozzo Canale ou Vincenzo Re (Cantone, 1992). Ces architectes ont puisé leur génie pour travailler en tant qu'architectes concepteurs, décorateurs, constructeurs, avec une approche assez similaire à celle 
adoptée lorsqu'ils travaillaient sur les architectures de pierre. Et il ne fait pas de doute qu'ils se servaient de la mise en scène comme laboratoire d'expérimentation du langage, de la forme et des solutions spatiales à utiliser dans cet immense travail de reconstruction qui, après le tremblement de terre de 1688 , était déjà en cours dans la ville et allait marquer de larges pans de son image actuelle. Il n'est pas seulement question de la libération des forces créatrices - au sens où l'entendait Piranèse - mais aussi au sens de l'évolution générale de l'architecture dans sa forme visible, c'est-à-dire de sa régulation et de son embellissement (Oechsin \& Buschow, 1984, p. 80)

17 Évidemment, il nous reste très peu de documents sur les architectures éphémères en dehors des représentations picturales et descriptions détaillées qui témoignent et représentent également le moyen de publicité le plus adéquat pour exprimer l'invention et l'idée architecturale. La perte de ces documents n'est pas seulement due au fait qu'elles étaient nées pour être détruites d'une manière spectaculaire, mais aussi au fait qu'à partir de la deuxième moitié du XVIII ${ }^{\text {e }}$ siècle l'ensemble ornemental de la fête approche de son déclin suite à l'avènement de la pensée des lumières et au mûrissement de la pensée sociale. Ce déclin implique la mise en accusation de tout l'imaginaire baroque et ce n'est qu'à partir de la deuxième moitié du $\mathrm{XX}^{\mathrm{e}}$ siècle que l'on commence à prêter davantage attention à ce courant de créativité, en reconnaissant enfin son importance indiscutable dans le développement de la ville. C'est précisément sur ce dernier point que j'entends insister: comment cette expérience s'avère-t-elle particulièrement utile pour notre réflexion sur la naissance de l'idée de place et des dynamiques des usages collectifs qu'elle anime?

Tout au long du XVII ${ }^{e}$ siècle on assiste, à Naples, au lent processus de transformation du largo - petite place carrefour - en piazza - place. Cette transformation ne se calque pas sur les espaces débordants des places romaines: pas de plan d'ensemble comme c'est le cas pour le réaménagement de la Rome papale. Dans un tissu urbain très dense et depuis toujours congestionné qui pendant des siècles ne s'est accru que sur lui-même, plutôt qu'élément de séparation entre îles et agglomérations d'habitation, la place devient ici un nœud de liaison à la fonction organisationnelle ainsi qu'un pôle centripète d'agrégation de fonctions collectives en relation avec les activités commerciales, les rencontres, les séditions, les fêtes, le spectacle théâtral (Cantone, 1992, p. 195-212). La modernisation de l'espace urbain se fait à travers de laborieuses acquisitions et soustractions du tracé romain, avec une fréquence rythmée par les fêtes et la mise en scène provisoire perçue justement en tant qu'occasion d'intervention et modification de l'aménagement urbain. Cette mise en scène éphémère représente en effet une première possibilité de réaliser une bonne partie de l'appareil décoratif, de l'aménagement spatial et de l'articulation de la place, comme si la ville même désirait mettre à l'essai son aménagement à travers une solution encore provisoire.

19 A l'occasion des fêtes, la place se transforme en espace théâtral pénétrable, traversable, où le bidimensionnel des coulisses se transforme en un décor tridimensionnel qui n'est pas seulement beau à regarder, mais peut être touché, pris d'assaut - comme c'est le cas pour les fêtes de cocagne - et vécu d'une manière collective. Le concept même de place prend une dimension singulière que l'on peut aisément revivre de nos jours dans des endroits spécifiques de la ville où l'on respire encore cette atmosphère spectaculaire de la mise en scène du quotidien avec ses rituels et ses pratiques de survivance. C'est le cas des zones destinées au marché comme par exemple Porta Nolana ou Porta Capuana. Ces zones ont conservé leur ancienne nature de zone de marché, de "vécu urbain", avec une 
vocation toute particulière pour le marché du poisson. Aujourd'hui, des nouvelles interprétations du sens des lieux se manifestent dans un contexte urbain qui, chaque jour, invente et met en scène des stratégies et des dispositifs de survie individuels et collectifs. Toutefois, le marché est presque demeuré semblable à ce qu'il était et les manières de faire des citadins en ce lieu sont caractérisées, aujourd'hui comme hier, par un sens du provisoire. La tendance à l'autoproduction (par exemple dans les systèmes de signalisation, information et direction), l'habitude de l'appropriation symbolique ainsi que physique de l'espace collectif en tant qu'espace fortement personnalisé, la gestualité qui devient un rituel dans l'aménagement quotidien de la scène urbaine se sont transformées en autant de catégories d'une façon collective de penser l'espace urbain.

Le théâtre donc, occupe un espace significatif dans la vie sociale de la ville de Naples et probablement c'est dans cette même dimension de théâtralité diffuse, dans laquelle l'éphémère se manifeste de manière provisoire face aux solutions formelles, aux langages et aux rituels d'utilisation de l'espace public, qu'on peut retrouver la spécificité de la déclinaison napolitaine de l'éphémère, qui, par de nombreux aspects, perdure dans le temps, bien après la fin même de la saison de la fête baroque.

21 Mais si on regarde la ville Naples du XVII ${ }^{e}$ siècle, ce n'est pas tout : ici, dans le processus de transformation de la ville, la transposition du langage de l'éphémère en architecture est évidente. Les appareils de la fête religieuse et les mécanismes complexes pour les processions fabriqués avec fleurs, cire, bois et carton-pâte (il en reste une trace dans les célèbres Gigli - lys de Nola) «tombent », pour ainsi dire, sur la dignité du marbre et de la pierre des magnifiques flèches de Cosimo Fanzago qui décorent les places principales de la ville du XVII ${ }^{e}$ siècle. Le thème de l'eau, élément fondamental pour combattre le feu redouté et exorcisé par la ville grâce à sa tradition centenaire des feux d'artifice, trouve son expression artistique dans les fontaines qui réinterprètent le thème de l'arc triomphal typique des appareils pour les fêtes commémoratives des victoires et du triomphe du prince (Cantone, 1992, p. 226-237).

Ainsi l'architecture de fête devient-elle champ d'expérimentation de l'architecture construite, donnant ainsi la preuve de son utilité et de sa contribution au progrès de la ville. C'est ainsi que les flèches et les fontaines occupent la place (elles ne l'engendrent pas comme cela arrive à Rome); elles occupent un espace préexistant en témoignage de l'appropriation définitive de l'espace rituel de la fête. Un cas exemplaire est celui de la petite Piazza Riario Sforza, la place où par un véritable coup de théâtre, le 16 décembre 1661, l'on dévoile la flèche de San Gennaro qui est offerte à la ville comme un petit joyau de l'espace. Ici, au milieu de gradins, escaliers et façades parées pour la fête, le plateau d'un petit théâtre en plein air est monté. 
Illustration 5 : Carré Riario Sforza, avec la Guglia de San Gennaro, Naples, réalisée par Ferdinando Sanfelice, 1661

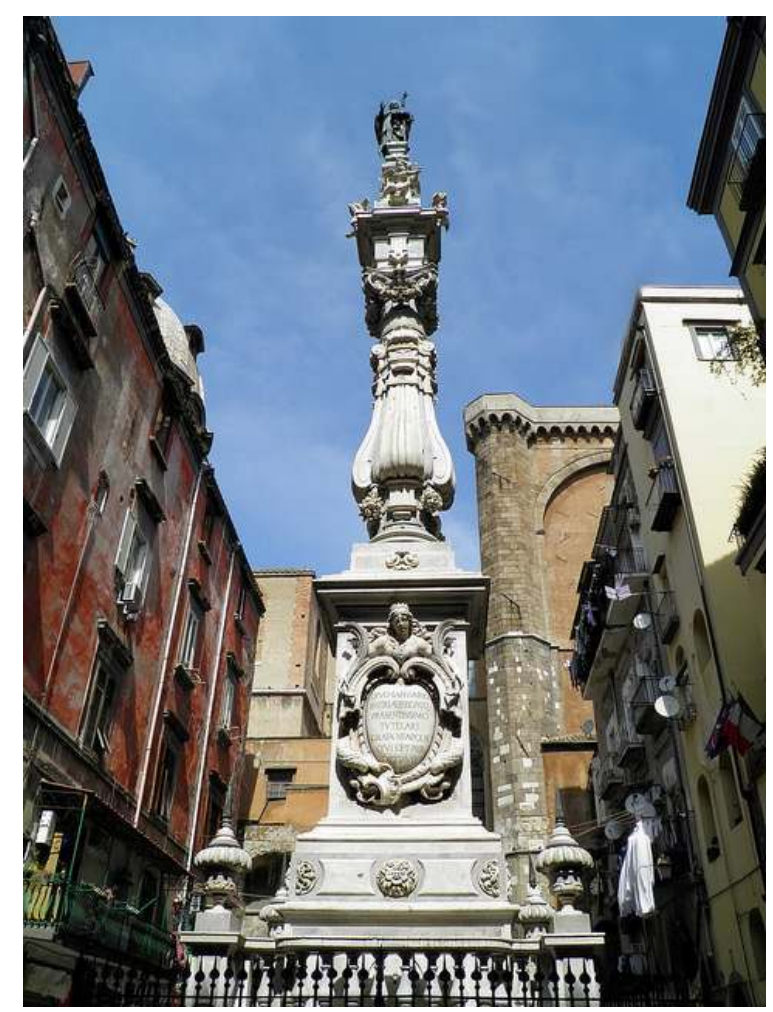

Source et copyright : (c) Alessandra Cirafici.

Illustration 6 : Carré de San Domenico Maggiore, avec la Guglia de San Domenico, Naples, réalisée par Domenico Antonio Vaccaro, 1666

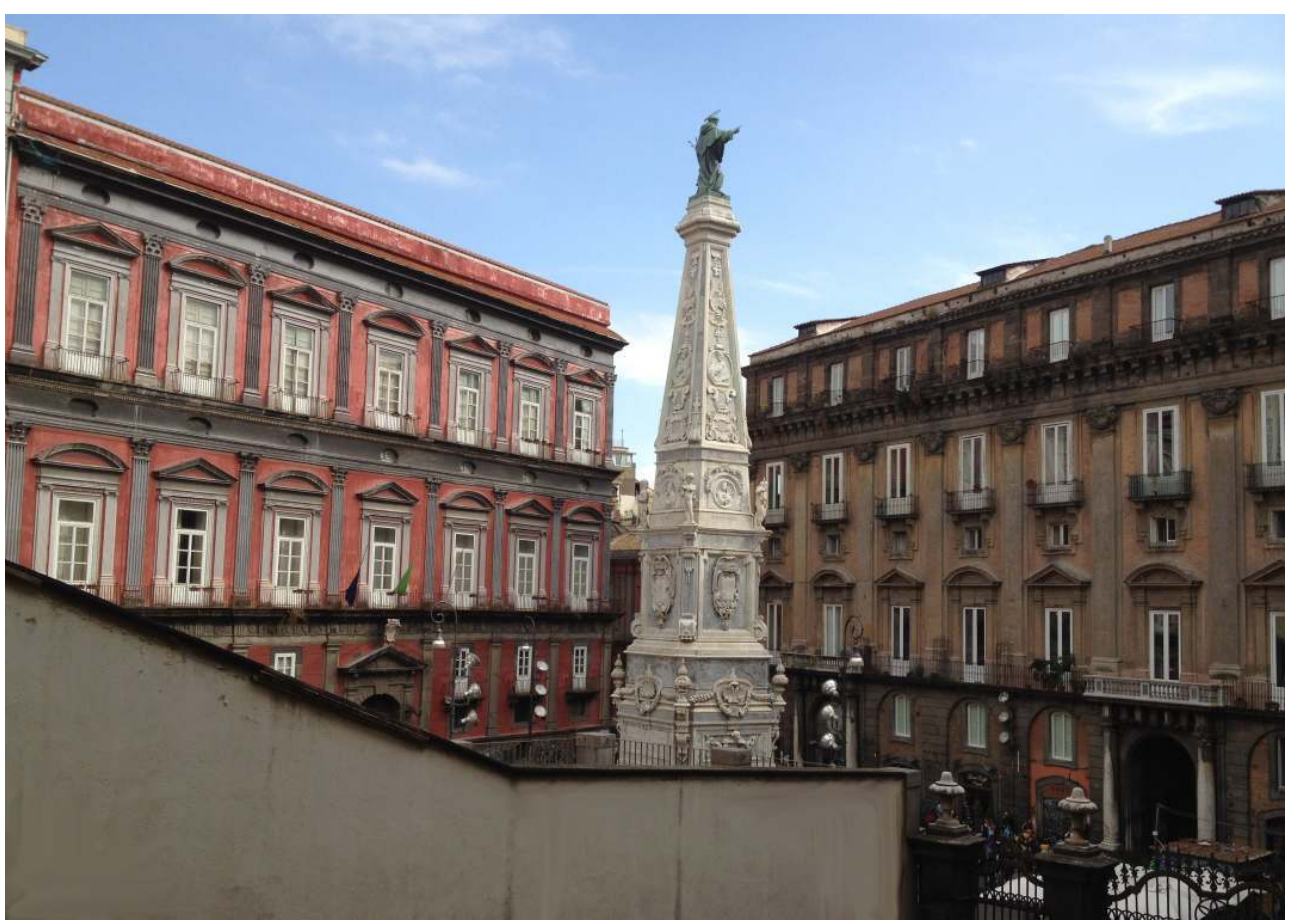

Source et copyright : Photo Salvatore Marino. Libre de droits. 
Illustration 7 : Carré du Gesù Nuovo, avec la Guglia dell'Immacolata, Naples, réalisée par Giuseppe Genoino, 1747

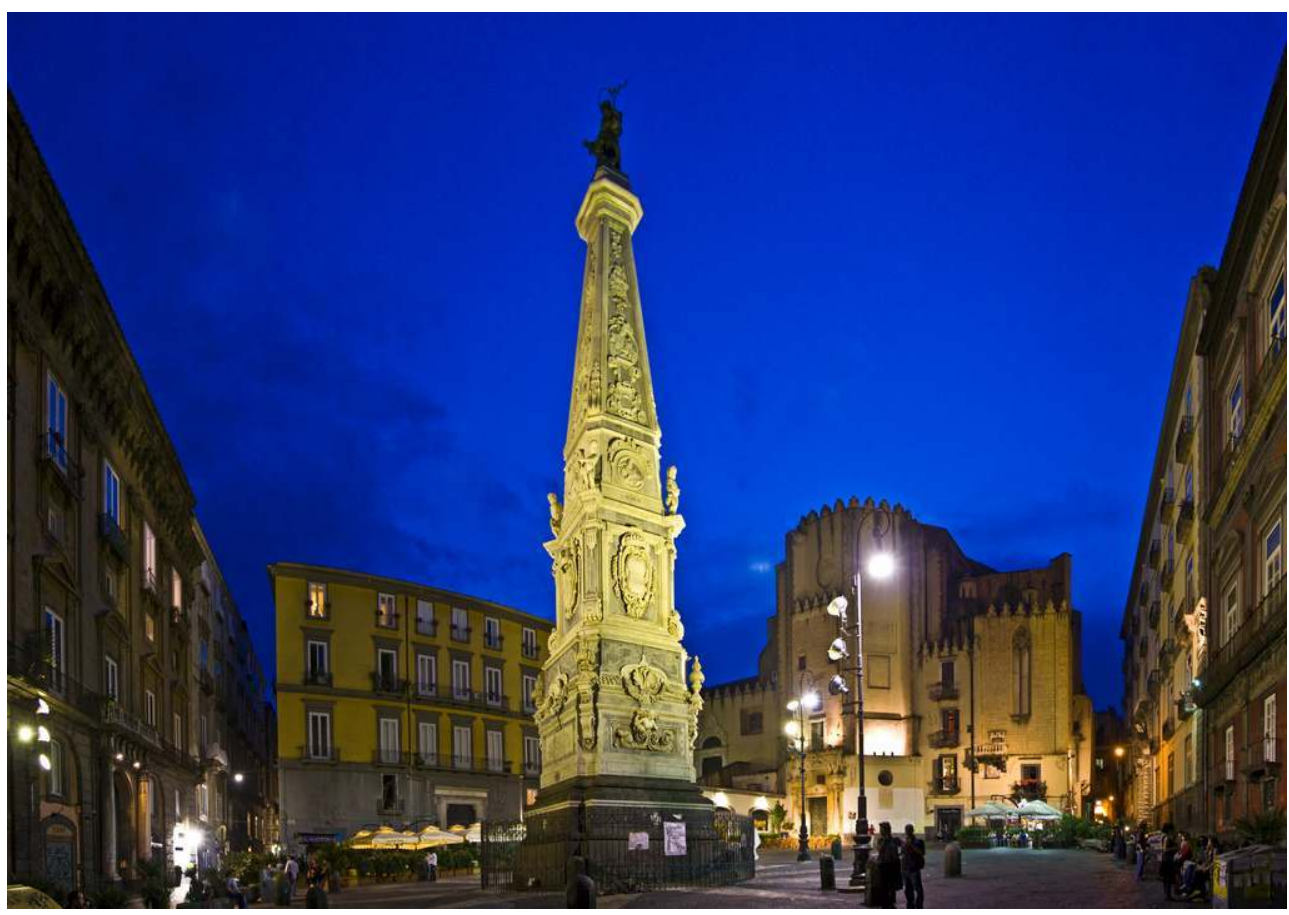

Source et copyright: Photo Stefen Geens, 29 octobre 2011. Libre de droits.

Et pendant que, par une compétition insolite entre les ordres monastiques, les flèches de San Domenico (signées par Domenico Antonio Vaccaro en 1666), de San Gaetano (signées par Cosimo Fanzago en 1679), de l'Immacolata (signées par Giuseppe Genoino en 1747), s'approprient la place suivant le même rituel, tout le lexique ornemental des décors provisoires se pétrifie et finit par déboucher sur la redéfinition des façades des palais nobiliaires. Des portails démesurés en piperne (la pierre du volcan) donnent sur des rues étroites dans une sorte de "hors-échelle » au point qu'ils semblent presque dépasser en dimension l'absence de raccourci, suscitant surprise et émerveillement. Les drapés, les cartouches, tout l'inventaire des décors éphémères de la fête se cristallisent sur les façades et sur leur double, allégorie de la consolidation graduelle des appareils de la fête (Salvatori \& Menzione, 1985). Entre temps, à l'intérieur des cours, conçues au même moment comme espaces privés et comme espace urbain et donc, lieu de performance collective, des toiles de fond théâtrales aux partitions spatiales dynamiques s'ouvrent avec une aisance hardie: il s'agit des spectaculaires escaliers ouverts, conception acrobatique au moyen de laquelle San Felice arrive à maîtriser les dénivellations de la rue Vergini, tout en inaugurant de nouveaux espaces consacrés à la fête et au théâtre: solutions spatiales surréelles, audacieuses au point de sembler inconscientes, imprégnées de cette audace qui n'appartient qu'à ce qui se sait provisoire (Gambardella, 1974).

Naples baroque se présente donc comme un joyeux et fastueux théâtre de rituels : dans les églises, les rues, les places et les palais nobiliaires. L'étroitesse de ses rues et de ses places et la nécessité de faire face à cette difficile condition spatiale devient une occasion de renouveau, le langage allégorique de la machine de la fête devient une stratégie de projet où, à travers une sorte d'oxymoron, le grand et le petit, l'intérieur et l'extérieur, le plein et le vide, le précaire et le permanent se confondent, se juxtaposent et se 
mélangent. La répétition obsédante de l'art, des activités en tout genre et des pratiques ludiques s'entremêlent dans un processus de remise en valeur et de nouvelle attribution de sens qui donne à l'expérience existentielle de l'espace une valeur anthropologique dont la trace est encore vivante. C'est l'espace de la fête dans une interprétation qui conduit à sa signification universelle.

Les fêtes ont marqué, en tout temps et en tout lieu, le besoin de la multitude passionnée, et ce dernier prend sa source d'abord dans la recherche des sensations et dans cet instinct précieux de la sociabilité qui porte les hommes à se réunir, à communiquer leurs craintes ou leurs espérances, leurs peines ou leurs plaisirs. (Chaussard, 1801, p. 21)

Illustration 8 : Ferdinando Sanfelice, Palazzo dello Spagnuolo, Naples, 1738 : I'escalier spectaculaire ouvert, appelé « aux ailes de mouette »

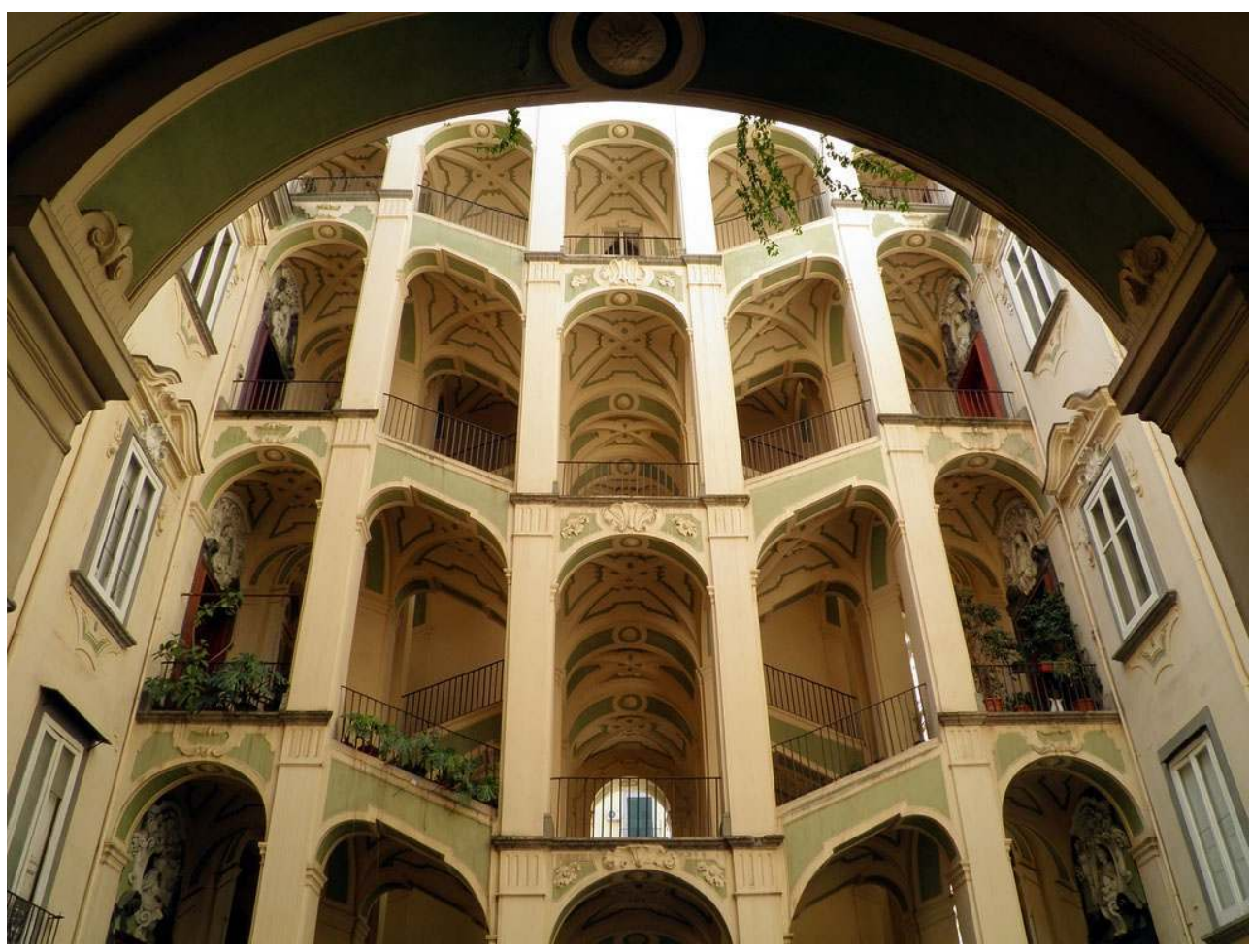

Source et copyright : ( $)$ Alessandra Cirafici

\section{Largo di Palazzo : un espace urbain en quête d'identité}

Nous voudrions, enfin, attirer l'attention sur le cas d'un espace qui, mieux que tout autre, a été le protagoniste de la fête à Naples, pour mieux comprendre ce qu'il est devenu. Il s'agit du Largo di Palazzo, à savoir l'actuelle Piazza del Plebiscito qui, dans l'imaginaire collectif, figure avec le golfe et le Vésuve parmi les icônes de la ville. Le Largo di Palazzo a été le lieu le plus extraordinaire de la fête baroque. Il en existe de magnifiques gravures le représentant lors de plusieurs occasions de fête, avec des décors incroyablement exubérants.

Pour prolonger notre réflexion, nous pouvons retracer les événements qui ont marqué l'histoire de ce lieu ainsi que les différentes manières dont il a été utilisé par la collectivité jusqu'à nos jours. 
Illustration 9 : Le Largo di Palazzo à Naples pendant le XVIIe siècle : la "Cocagne " réalisée par Ferdinando Sanfelice à l'occasion de la naissance de l'Infantareale, 1740

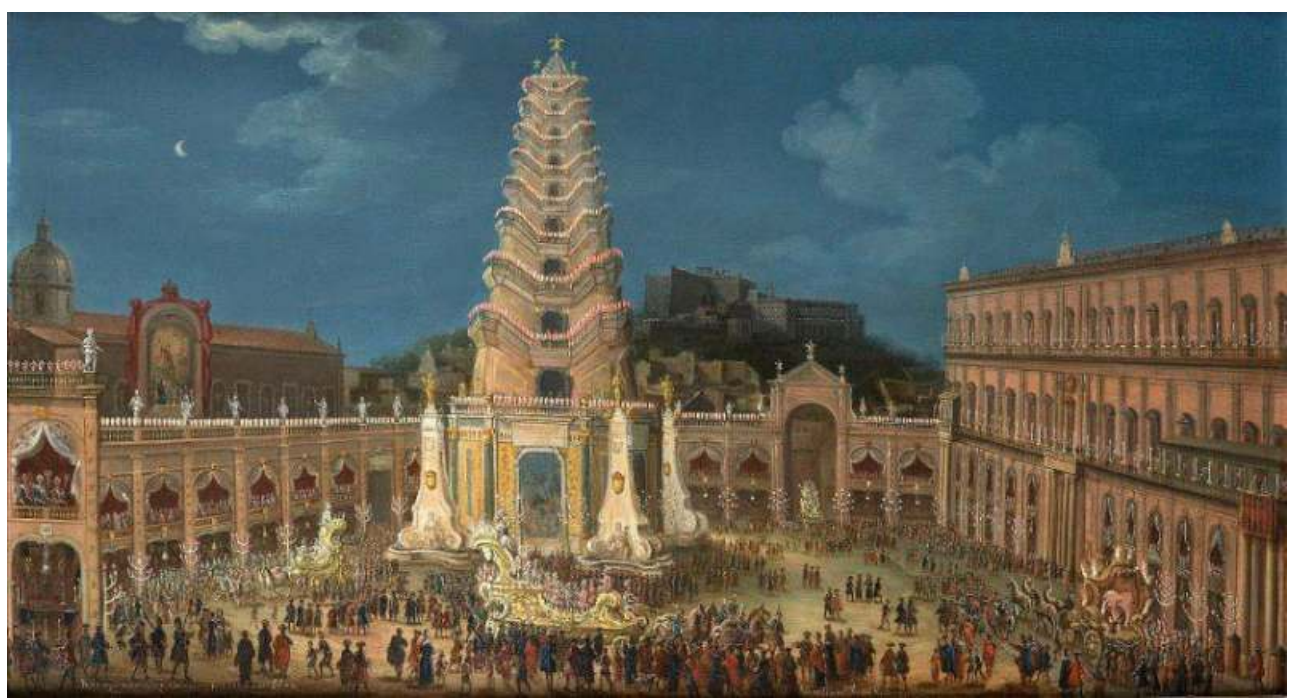

Source et copyright: Tommaso Ruiz, huile sur cuivre, collection privée. In AA.VV. Capolavori in Festa Effimero Barocco a Largo di Palazzo, Napoli, 1997, p. 174. Avec l'aimable autorisation d'Ermanno Bellucci (Sovrontendenza Acheologica e curatore del catalogo).

Illustration 10 : Largo di Palazzo avec Cocagne, 1700. ca

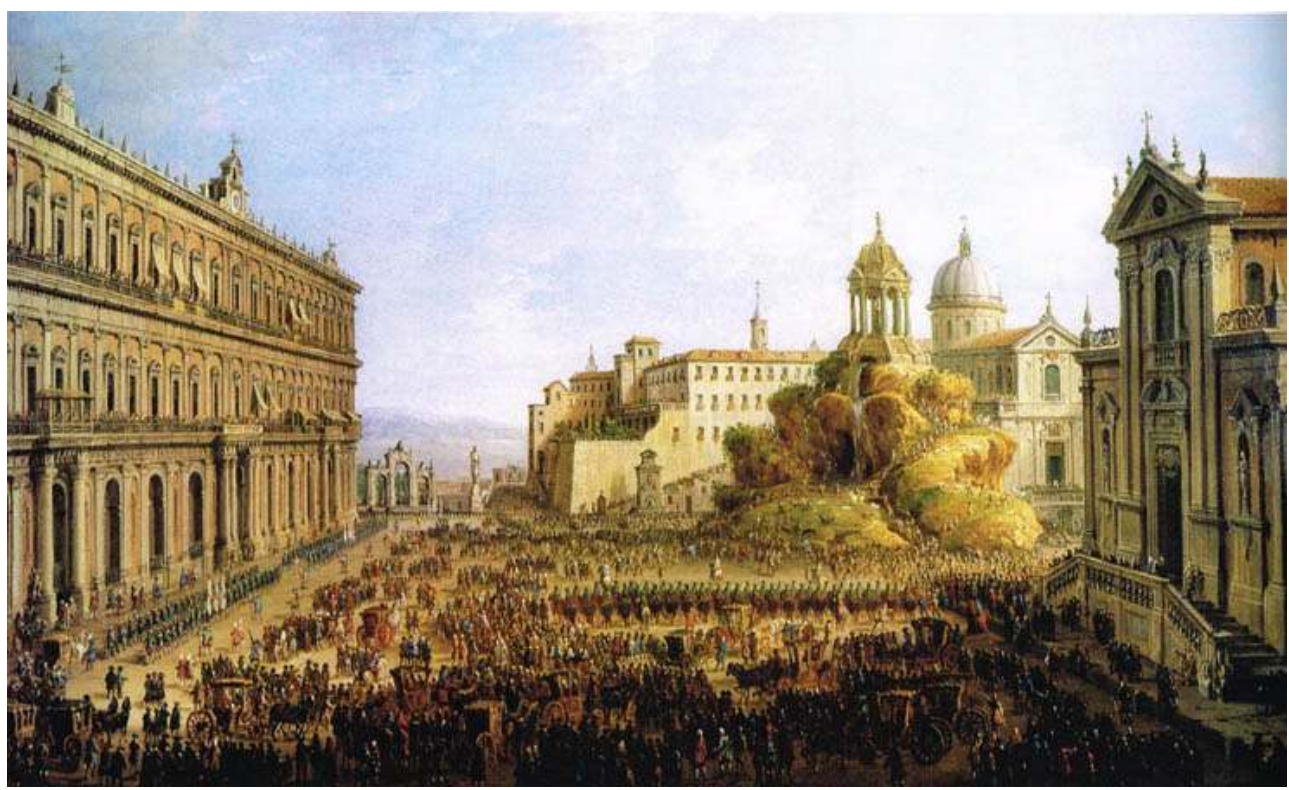

Source et copyright : Antonio Joli, huile sur toile. Beaulieu, The Lord Montagu of Beaulieu, Palace House. In AA.VV. Capolavori in Festa Effimero Barocco a Largo di Palazzo, Napoli, 1997, p. 175. Avec l'aimable autorisation d'Ermanno Bellucci (Sovrontendenza Acheologica e curatore del catalogo).

Tout au long de la période comprise entre le XVII et le XVIII ${ }^{e}$ siècle, le Largo di Palazzo avec ses rues alentour représentait le lieu par excellence où se déroulait le rite quotidien de l'exhibition de soi en dehors de sa propre habitation. Il représentait le noyau cérémonial de la ville, l'espace où confluaient toutes les classes sociales, du menu peuple à la haute aristocratie, à la cour ; c'était le miroir de l'identité de la ville et du Royaume et le lieu de l'union idéale entre peuple, culture, culte et religion. Le lieu où la fête dépassait 
le domaine restreint de la tradition de cour et s'intégrait à la vie quotidienne, d'une façon générale, support de la félicité publique.

Toutes les manifestations qui s'y déroulaient avaient pour objectif de recomposer l'unité sociale, de confirmer la fiabilité du gouvernement et sa maîtrise de la plèbe, de célébrer le prince. Mais c'était aussi, voire avant tout, le lieu destiné à révéler les hiérarchies sociales. Tout le quartier foisonnait de résidences de nobles qui montraient leur rang depuis les balcons de leurs palais pour participer à la fête comme s'ils étaient dans les loges d'un théâtre. Dès cette époque, la ville se présentait aux étrangers pleine de monde, encombrée de carrosses et caractérisée par un frénétique va-et-vient (Naples a été la première ville d'Europe à introduire le trottoir conçu en tant qu'espace où la plèbe pouvait admirer le passage des carrosses!). La vitesse et le mouvement exprimaient une envie de nouveauté et de modernité que la ville voulait afficher. La fête faisait évidemment partie de cet étalage collectif, elle était justement articulée afin que des morceaux de messages diversifiés puissent atteindre les différentes couches de la société : allégories savantes tirées de la littérature - que la plupart des citoyens n'arrivait même pas à comprendre - destinées à un public cultivé, fêtes de cocagne et spectacles pyrotechniques adressés au menu peuple. Toutefois, les différents messages étaient captés dans un système complexe de communication qui surmontait toute barrière sociale, un langage qui s'adressait à tous et était en mesure d'exprimer tant les rapports de pouvoir que les aspirations, les sentiments, les tendances du moment. Donc, un véritable instrument pour gouverner le peuple (d'où prend son origine le célèbre dicton Festa Farina e Forca - Fête Farine et Fourche !) et en plus de cela, il engendrait lui-même de nouveaux liens, de nouvelles relations, des accords et des désaccords... Une caractéristique plus générale de la fête, bien expliquée par Gadamer quand il dit :

Lorsque quelque chose est liée à l'expérience de la fête, c'est ceci qui empêche tout isolement de l'un par rapport à l'autre. La fête est communion et représentation la plus haute de la communauté. La fête est toujours destinée à tous. (Gadamer, 1977, p. 52)

Il n'est donc pas étonnant que des architectes réputés aient été impliqués dans les décors de ces manifestations qui étaient largement diffusés dans de nombreuses publications afin d'expliquer les intentions des organisateurs et en garder la mémoire au fil des ans. Parmi les manifestations qui avaient lieu dans l'espace du Largo di Palazzo, en dehors de celles liées aux naissances, mariages et triomphes de la maison royale, il faut bien sûr mentionner le Carnaval, une fête singulière, qui se situait entre le rituel religieux et la fête civile. Pendant cette période, comme chacun était en quelque sorte autorisé à bouleverser les règles du quotidien, il était plus facile d'évaluer le consentement au pouvoir ou le degré de malaise du peuple (Lattuada, 1997).

31 Toutefois, bien qu'il ait été vécu comme un centre névralgique de la vie de la ville durant la fête, le Largo di Palazzo a eu beaucoup de mal à prendre sa physionomie d'espace urbain à proprement parler. Au fond, ce n'était qu'un terrain de terre battue qui s'étendait au pied de la colline de Pizzofalcone, l'acropole du premier établissement des Grecs, juste en face du Palais Royal. 


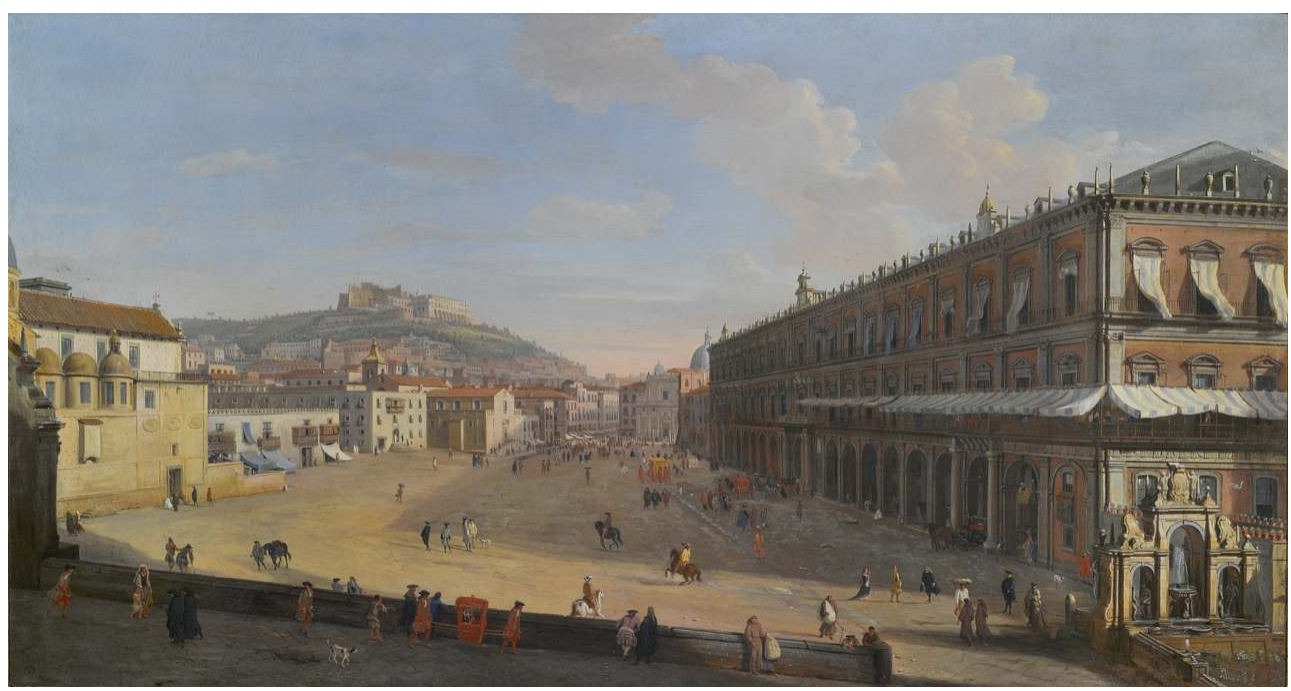

Source et copyright : Gaspar Van Wittel nommé Gaspare Vanvitelli (1653-1736, huile sur cuivre, collection privée. in AA.VV. Capolavori in Festa Effimero Barocco a Largo di Palazzo, Napoli, 1997, p. 144. Avec l'aimable autorisation d'Ermanno Bellucci (Sovrontendenza Acheologica e curatore del catalogo).

Ce qui semblerait expliquer le fait qu'il ne figure ni dans les minutieuses descriptions de la ville, ni dans les nombreux guides de l'époque, comme c'est d'ailleurs le cas pour bien d'autres endroits. Tout au long du XIX ${ }^{e}$ siècle, il a encore de la peine à prendre une physionomie de place et l'on pourrait même affirmer qu'il commence à y arriver au fur et à mesure qu'il perd sa connotation de théâtre collectif en plein air.

Néanmoins, il faut mettre en relief que la conformation urbaine définitive de cet espace ne semble guère différente par rapport aux nombreux décors qui l'avaient occupé au fil des ans. L'idée du grand hémicycle muni de colonnes voulu par Joachim Murat au centre duquel se situe, avec ses trois coupoles, la Basilique de San Francesco di Paola érigée au début du XIX ${ }^{e}$ siècle à la demande de Ferdinand IV, avait été en effet proposée plusieurs fois pour les décors éphémères de la fête baroque. 


\section{Illustration 12 : Plans de foires}

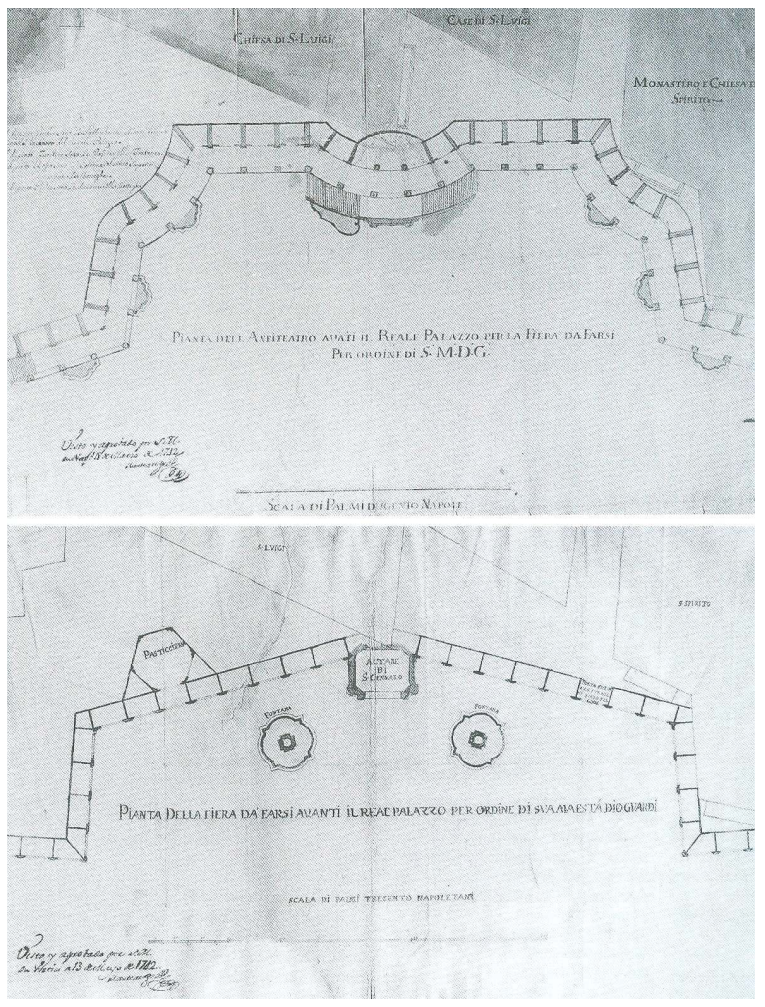

En haut : Plan d'une foire Largo di Palazzo. En bas : Plan d'une foire qu'on devait organiser en face du palais royal par ordre de S.M. Dio Guardi. 13 mai 1742.

Source et copyright: Naples, Archives de l'Etat. Plans et Dessins. Cart. XXV n 3 (en haut) et cart. XXV $n^{\circ} 70$ (en bas). Avec l'aimable autorisation d'Ermanno Bellucci (Sovrontendenza Acheologica e curatore del catalogo). 


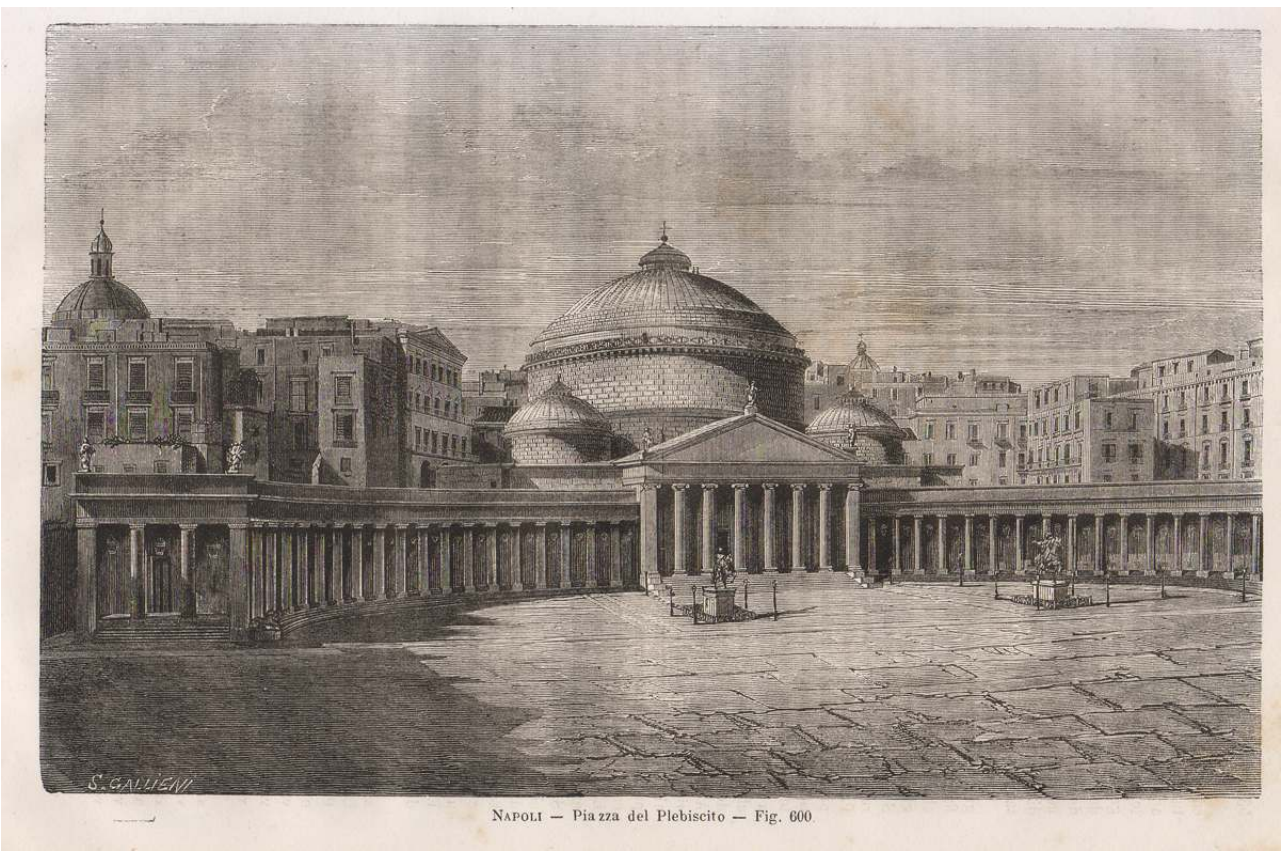

Xilographie $220 \times 140 \mathrm{~mm}$.

Source et copyright : Dizionario corografico dell'Italia Amato. Milano : Vallardi editore. 1865. Avec l'aimable autorisation d'Ermanno Bellucci (Sovrontendenza Acheologica e curatore del catalogo).

Quelques dessins de projet en témoignent. Il semble que, finalement, le provisoire ait pris le dessus en se cristallisant à nouveau dans une forme définitive et pour ainsi dire incisive.

Toutefois, le lieu semble n'avoir jamais perdu son éclectisme qui lui a permis de prendre de multiples visages suivant les différentes performances collectives qui s'y sont déroulées. Au fil des ans, la place a continué à être le site des célébrations collectives et des rituels de la ville. C'était le cas des fêtes du peuple comme par exemple celle organisée à l'occasion de la réalisation de la fontaine du Serino - la rivière qui est reliée au premier aqueduc de la ville - en 1885 , avec son jet d'eau spectaculaire et d'une certaine manière cathartique. Mais c'était aussi le cas des grandes réunions de peuple lors de l'arrivée du Führer à Naples, ou lors du plébiscite de 1945 qui établit le passage à la République, ou encore à l'occasion des visites des papes, plus récemment à l'occasion de la commémoration de la mort de Pino Daniele, l'un des chanteurs italiens les plus aimés à Naples.

Intéressantes et suggestives sont aussi les images de la vie de la place au cours des années 1930 dans une dimension presque surréaliste d'espace public, lieu de l'action collective. 

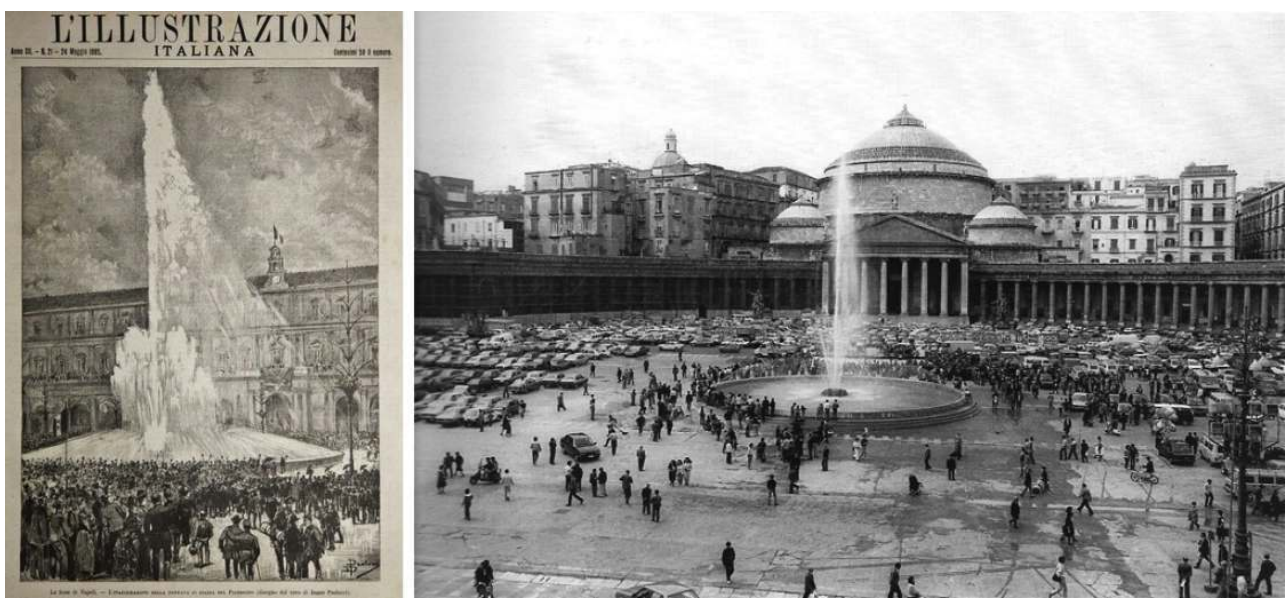

A gauche : Piazza del Plebiscito 1885, la Fête du Serino. A droite : Piazza del Plebiscito 1985, reproduction de la fontaine du Serino.

Source et copyright : A gauche : L'Illustrazione Italiana, anno II. 24 mai 1885. Trevesi : Ed. Fratelli, n² 21. A droite : Napoli : Archive de photos Parisio. Les ayants-droits de l'image peuvent se manifester auprès de l'auteure.

Illustration 15 : La place du Plebiscito pendant les années 1930
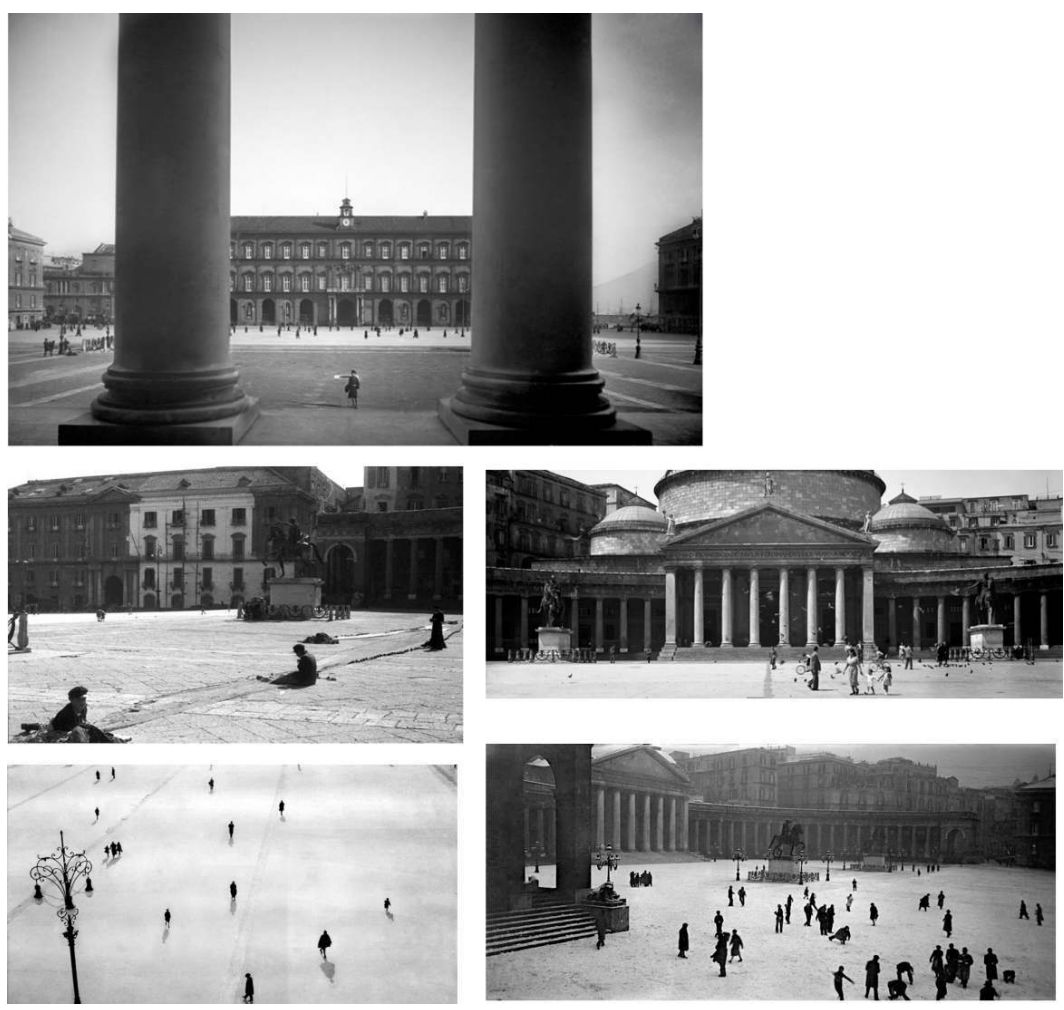

Source et copyright : Napoli : Archive de photos Parisio. Les ayants-droits de l'image peuvent se manifester auprès de l'auteure. 
Illustration 16 : La place du Plebiscito utilisée comme stationnement pour autos et bus pendant les années 1960

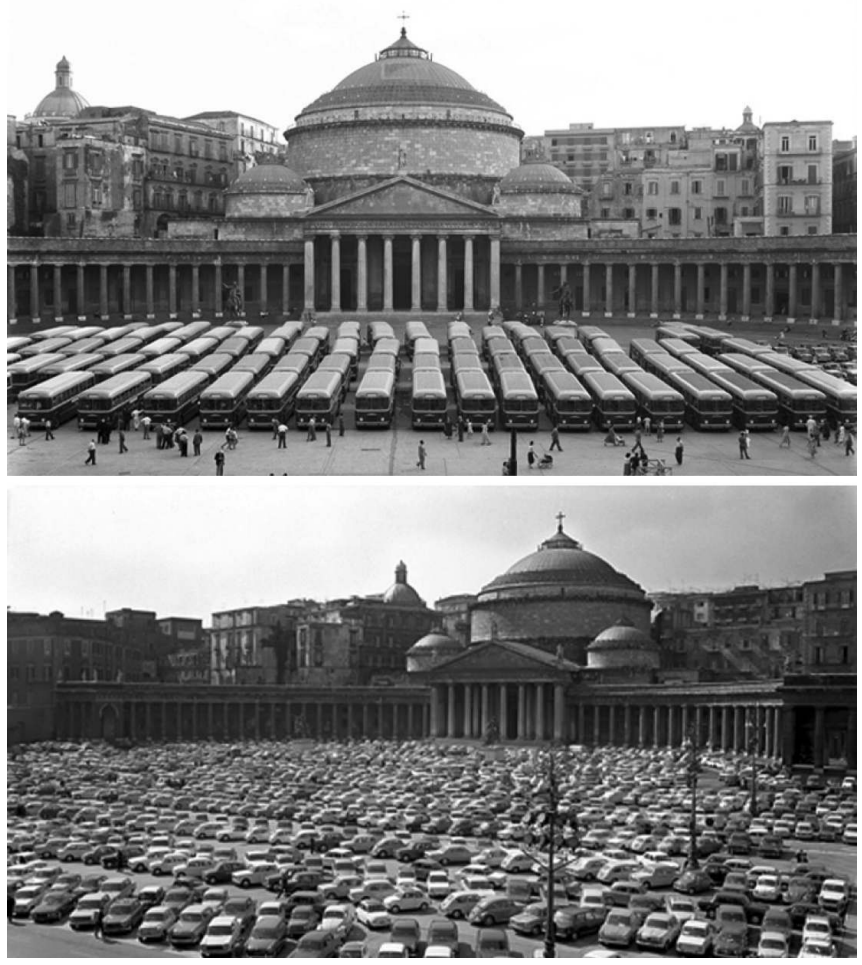

Source et copyright: Napoli : Archive de photos Parisio. Les ayants-droits de l'image peuvent se manifester auprès de l'auteure.

Surprenantes sont les images qui témoignent l'état d'une place transformée en parking jusqu'aux années 1980 dans une condition d'usage qui empêchait à l'espace d'interpréter son véritable rôle d'agrégation des individus.

Tout à fait différent est le rôle joué par l'espace de la place, quand, autour des années 2000, l'administration municipale, après avoir courageusement délibéré sur sa piétonisation, y fait ériger, à l'occasion des fêtes de Noël, des installations d'art contemporain. C'est le moment où la place a repris sa fonction de lieu de la fête en étant reliée à son usage traditionnel : en effet, les mises en scène proposées par des artistes célèbres comme Mimmo Paladino, Rebecca Horn, Richard Serra et Anish Kapoor, avec leurs constructions artificielles et éphémères dont le résultat n'est finalement qu'un bref moment d'étonnement, ont tout à coup réinterprété la place comme espace de la performance collective. L'art public souvent se propose comme dispositif perceptif qui demande l'interaction des spectateurs qui vont devenir acteurs de la mise en scène, exactement comme à l'époque de la fête baroque, et les exemples cités en sont une expression claire. 
Illustration 17 : Spiriti di madre perla (Mother-of-pearl Spirits)

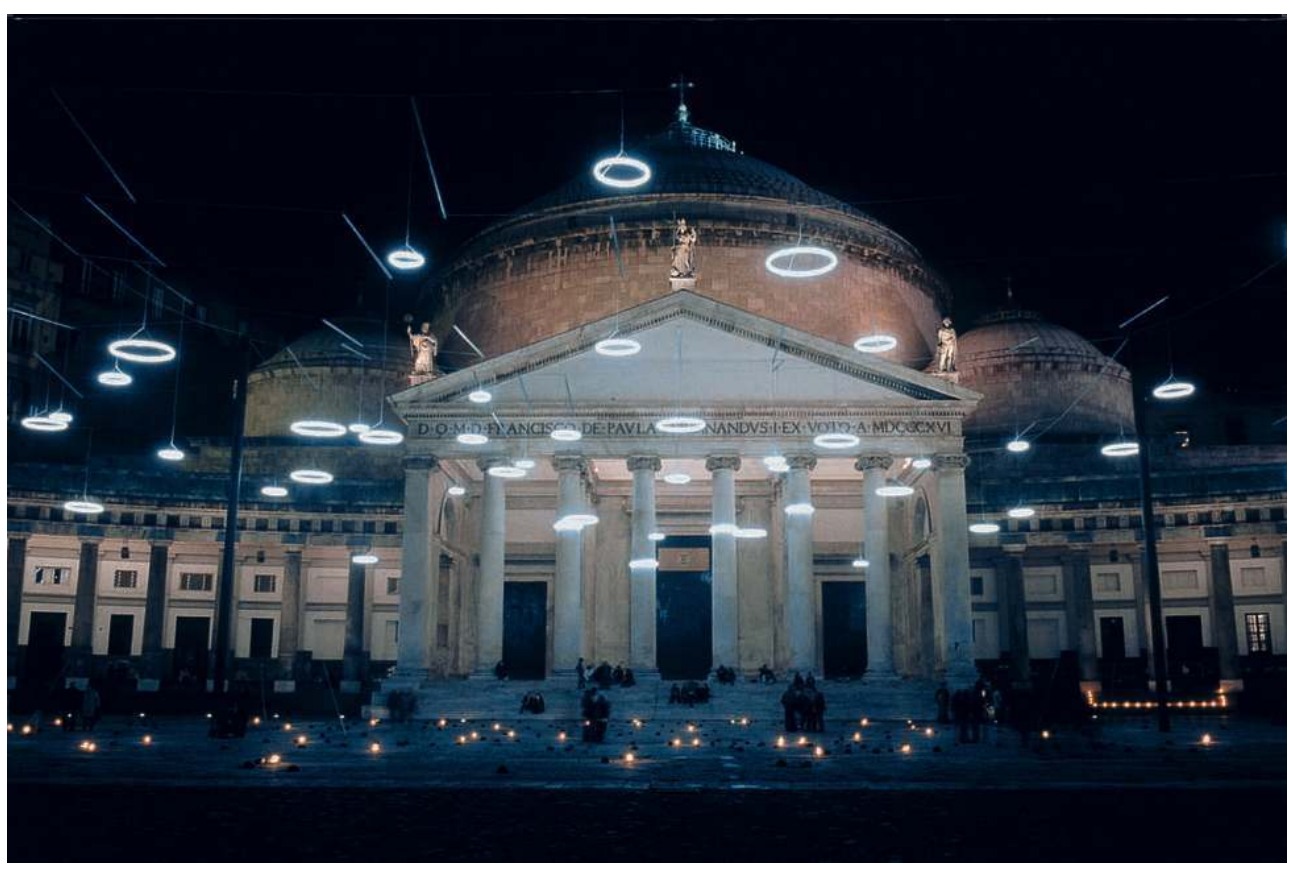

NAPOLI, PIAZZA DEL PLEBISCITO. 14/12/2002-31/01/2003, MUSIC BY HAYDEN CHISHOLM, TEXT BY REBECCA HORN. INSTALLATION RÉALISÉE PAR L'ARTISTE REBECCA HORN, DÉCEMBRE 2002.

Source et copyright: Photo Peppe Avellone. Libre de droits.

Illustration 18 : La montagna di sale

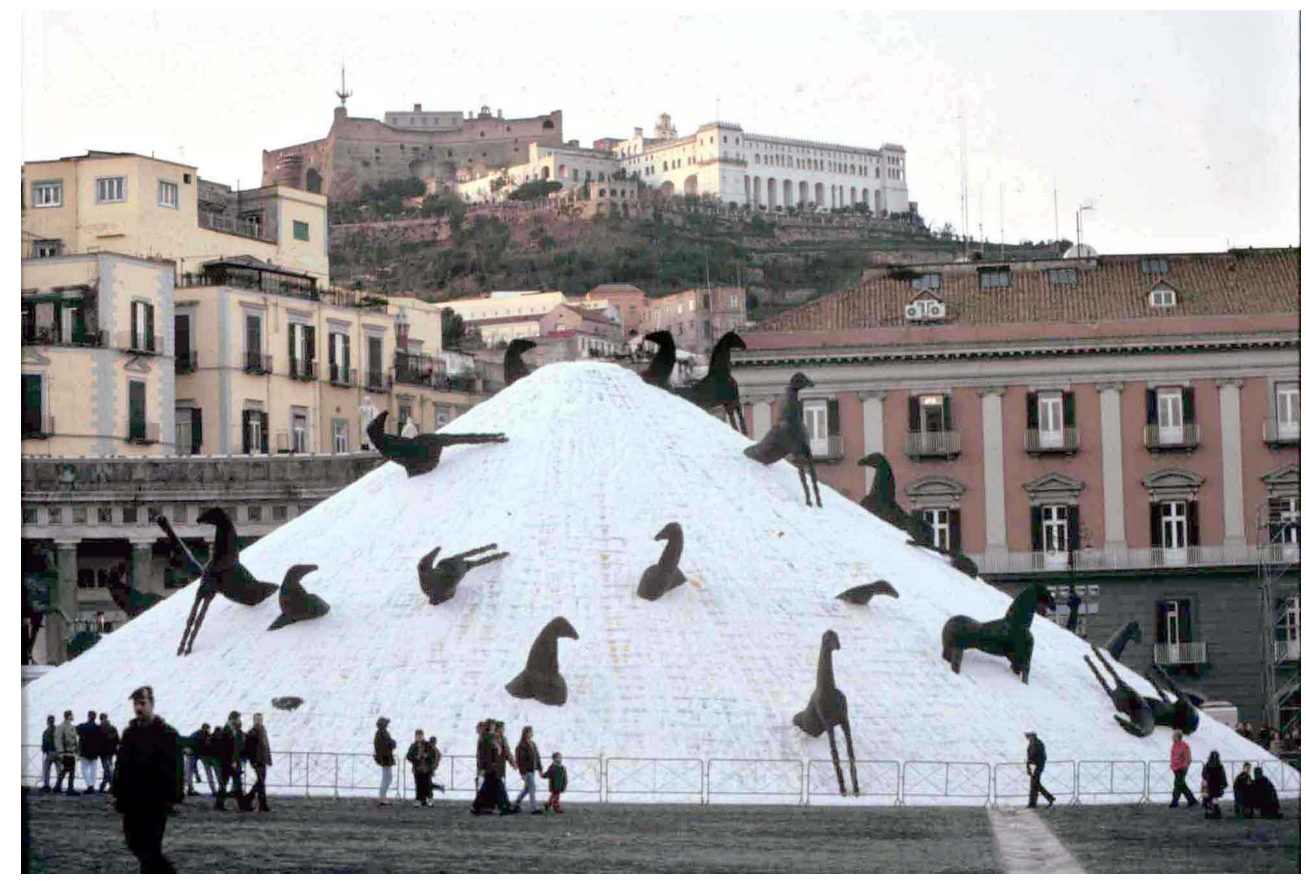

Napoli, Piazza del Plebiscito. Installation réalisée par l'artiste Mimmo Paladino, décembre 2011.

Source et copyright : [en ligne]. Disponible sur : http://www.artribune.com/ (consulté le 12 avril 2017). Libre de droits. 


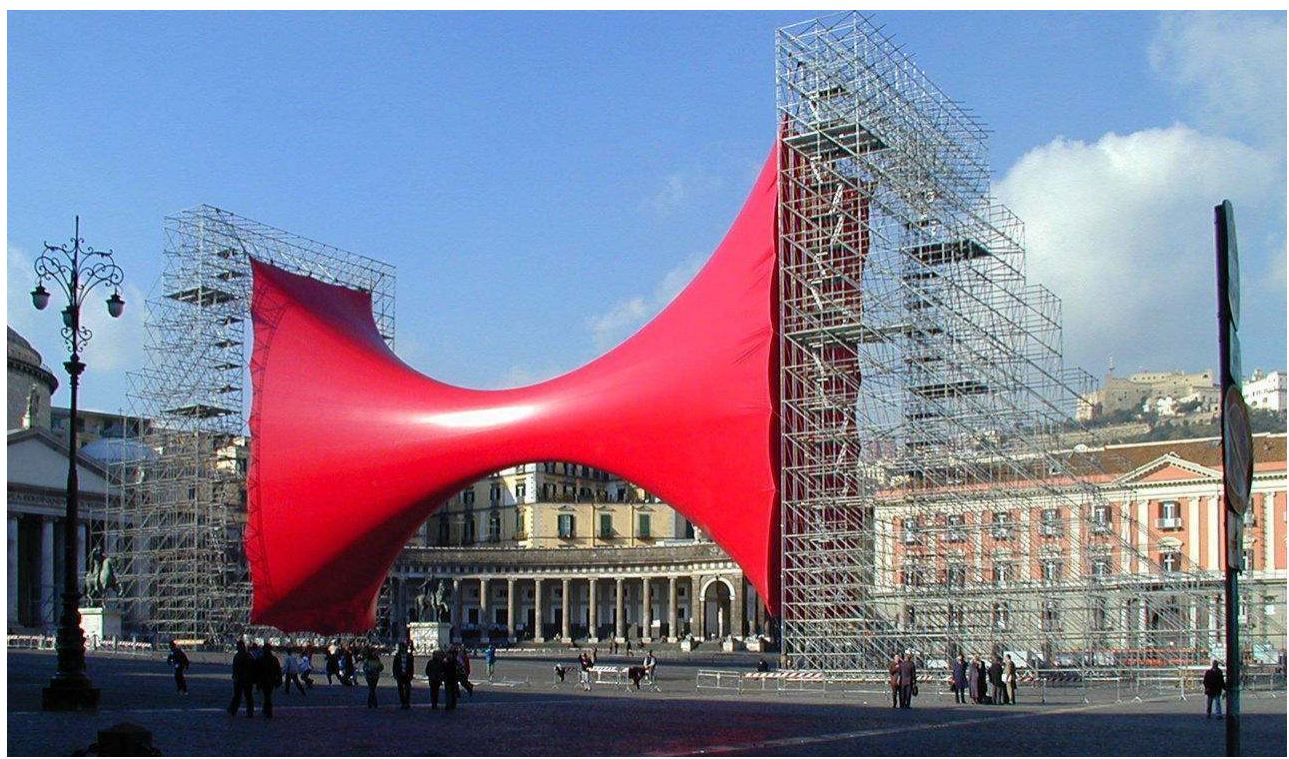

Napoli, Piazza del Plebiscito. Installation réalisée par l'artiste l'artiste Anish Kapoor, décembre 2001. Source et copyright : [en ligne]. Disponible sur : https://fotosintez.files.wordpress.com (consulté le 17 avril 2017). Libre de droits.

\section{Illustration 20 : Scultura "travolgente"}

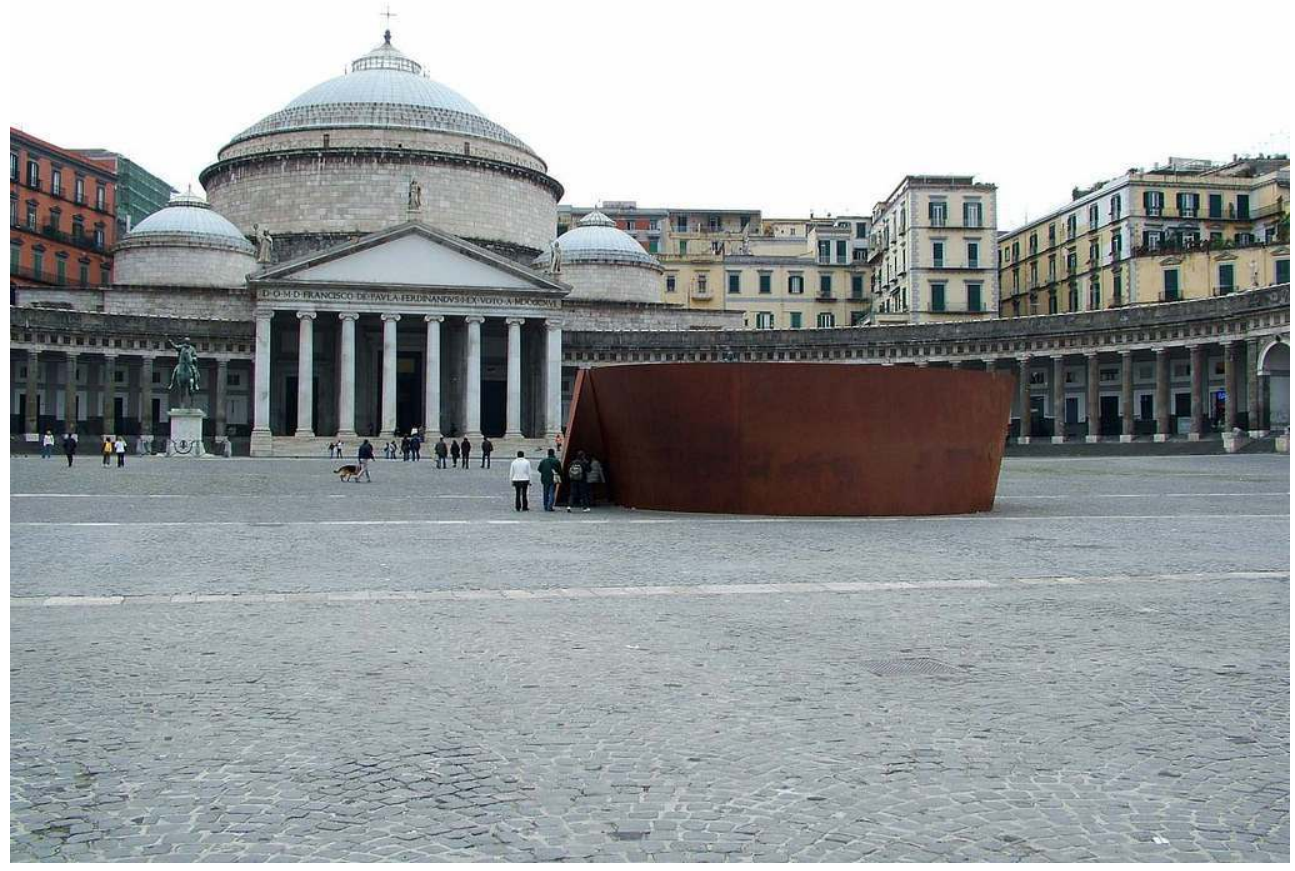

Napoli, Piazza del Plebiscito. Installation réalisée par l'artiste Richard Serra, décembre2004.

Source et copyright : [en ligne]. Disponible sur : http://www.adspazio.it/la-scultura-travolgente-richardserra/ (consulté le 17 avril 2017). Libre de droits. 
Illustration 21 : 5 janvier 2016, flash mob organisé pour la commémoration de la mort de Pino Daniele

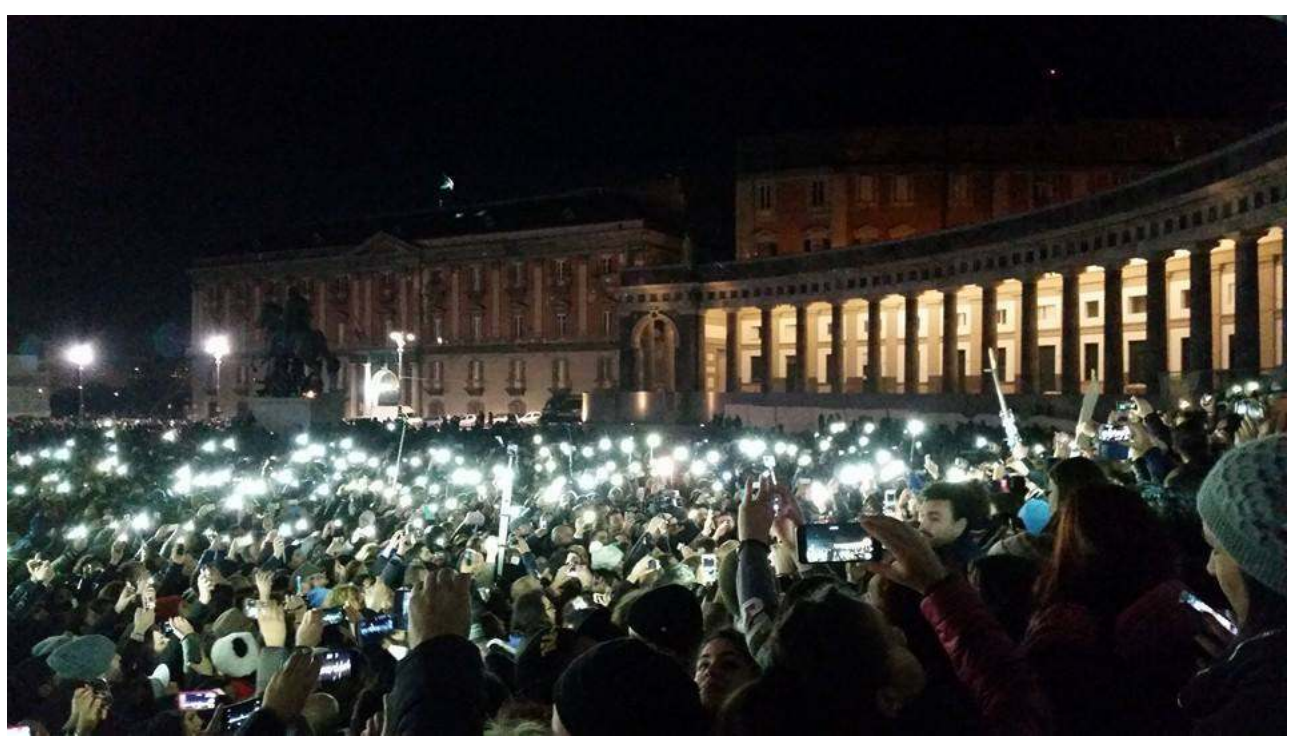

Source et copyright : [en ligne]. Disponible sur : http://www.napolike.it/pino-daniele-funerali-piazzaplebiscito-napoli/ (consulté le 17avril 2017). Libre de droits.

39 Aujourd'hui, à cause de la crise globale, on ne peut plus envisager des interventions d'art public d'une telle importance et donc, si l'on regarde l'une de ces images quelconques de la Piazza del Plebiscito, telle qu'elle se présente aujourd'hui, il est tout à fait évident que, dépourvue du décor éphémère de la fête, dans sa sobriété formelle même, elle nous apparaît comme une sorte de vide urbain absente à sa propre connotation spatiale. Comme si, une fois fermée à la circulation et à ses ravages, il n'y avait jamais eu de véritable projet pour le réseau des relations spatiales et des fonctions que l'on entendait y activer. On dirait une toile de fond... dont le concept n'a guère évolué par rapport à ce terrain de terre battue qui, au XVII e siècle, s'ouvrait au pied de la colline de Pizzofalcone, juste devant la façade du Palais royal, un espace en suspens... 


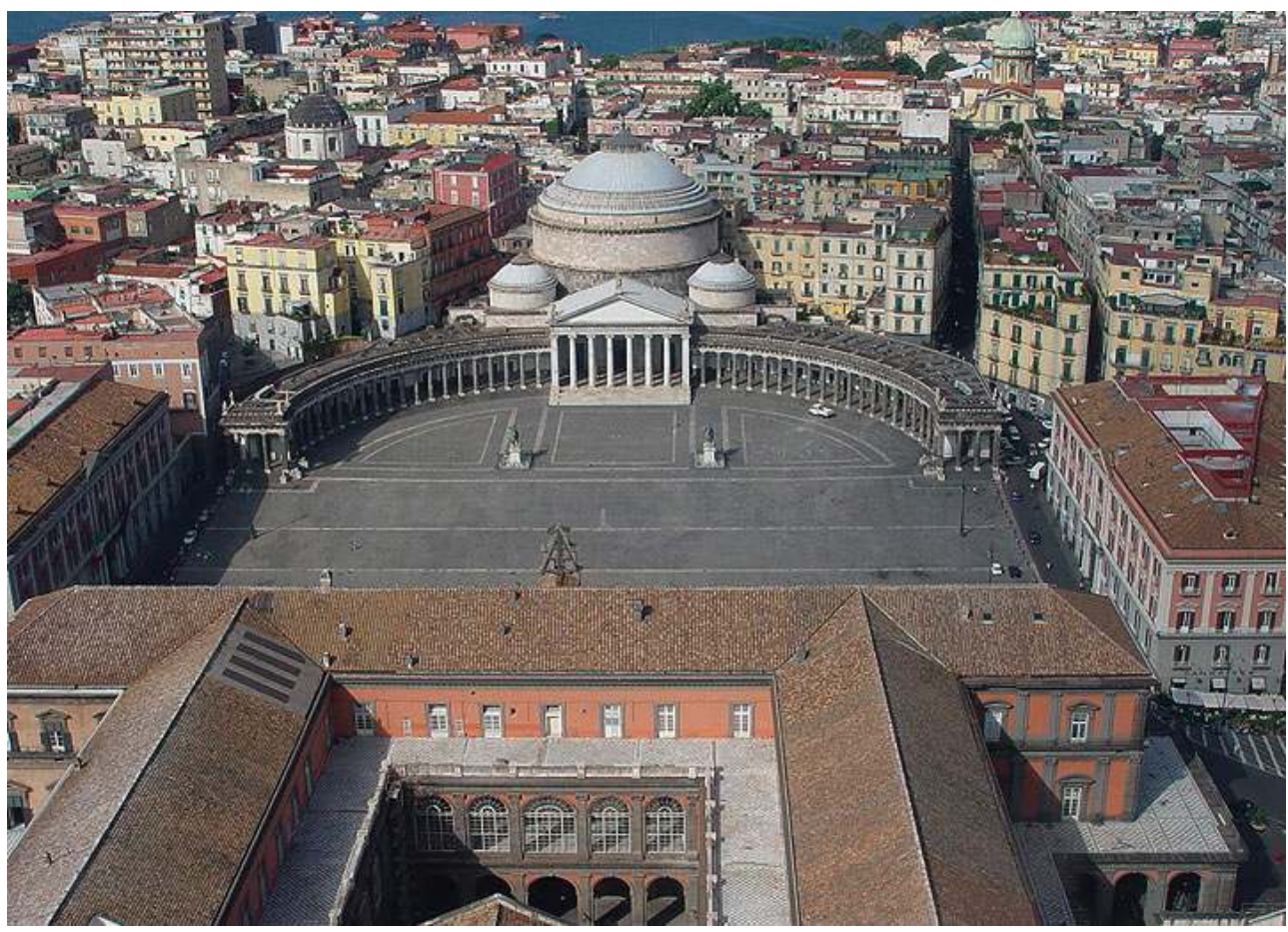

Source et copyright : [en ligne]. Disponible sur : http://napolinfoto.blogspot.com (consulté le 25 mars 2016). Libre de droits.

\section{Conclusion}

«Public est le mot magique qui circonscrit et doit circonscrire la question de l'architecture de fête » (Oechslin \& Buschov, 1984, p. 43). Mais «public » c'est la façon d'entendre aussi la notion d'espace - et notamment de l'espace de la ville conçue en tant que réseau de relations et donc, au sens large du terme, en tant qu'espace de vie - à savoir un intéressant enchevêtrement de signes, symboles, codes et métaphores de l'habiter. Un système complexe dont il est intéressant de saisir les signes distinctifs, afin de comprendre le sens de ces actions individuelles ou collectives ayant le pouvoir de modifier la structure physique et les composantes relationnelles de l'espace urbain, à travers un processus qui, souvent d'une manière spontanée, déclenche des stratégies de comportement grâce auxquelles l'habiter acquiert de nouvelles possibilités de sens.

Si aujourd'hui on a l'habitude de définir une performance urbaine comme une action par laquelle un ou plusieurs sujets attribuent une nouvelle signification à un espace en projetant une intervention qui vise à modifier l'état d'un lieu en altérant les paramètres de sa perception et en envisageant une utilisation inattendue ne semble pas apparemment adaptée à ses contours physiques (Campos, 2011), on peut bien affirmer que le théatre de la fête baroque est donc, incontestablement, une performance à part entière qui nous offre encore bien des suggestions à partir desquelles nous pouvons nous interroger sur la possibilité de projeter à travers l'éphémère. Il s'agit ainsi de réfléchir sur le pouvoir du provisoire qui peut encore donner naissance à des comportements qui, à leur tour, engendrent l'espace public. A ce sujet, il est intéressant de souligner la double fonction qu'avaient les citoyens pendant la fête : ils étaient des spectateurs, mais avant 
tout, des acteurs d'une gigantesque performance collective dont le pouvoir d'agrégation ne diffère guère de celui des flash mob modernes.

Rétrospectivement donc, la fête baroque revêt une grande importance pour la compréhension des fonctions publiques et urbaines, dont l'analyse ouvre aujourd'hui une compréhension de l'évolution de la ville historique par rapport à la ville contemporaine, et une interprétation du rôle de médiateur toujours joué par l'architecte.

Et dans ce domaine, il est intéressant de réfléchir à la possibilité de se servir du provisoire - tellement présent sur la scène de la ville contemporaine - en tant qu'accélérateur d'innovation, opportunité d'expérimentation et vérification, moteur du renouveau stylistique et comportemental, catalyseur de nouvelles relations spatiales. Dans ce cadre, le concept de macchina da festa qui continue de désigner l'aspect artificiel et éphémère de la construction temporaire, acquiert une signification supplémentaire : c'est le caractère temporaire, le renoncement conscient aux lois de la firmitas de l'architecture construite qui vient en aide de l'architecte et l'incite à inventer, aujourd'hui comme hier, l'espace de la ville.

\section{BIBLIOGRAPHIE}

Anceschi, Luciano. 1984. L'Idea del Barocco. Studi su un problema estetico. Bologna: Nuova Alfa Editoriale.

Assunto, Rosario. 1970. Leibniz e il Barocco. In: Barocco Europeo, Barocco Italiano, Barocco Salentino. Relazioni e communicazioni al Congresso internazionale sul Barocco, Lecce et Terra d'ottante, 21-24 septembre 1969. Lecce. p. 299-350.

Benjamin, Walter. 2005 [1963]. Immagini di città. Torino: Einaudi.

Campos, Carlos. 2011. La Performance Arquitectònica. Nuevas experiencias en la signification del Espacio Urbano. Buenos Aires: Bisman ediciones.

Blunt, Anthony. 1975. Neapolitan Baroque and Rococo Architecture. London: TBS The Book Service.

Cantone, Gaetana. 1984. Napoli Barocca e Cosimo Fanzago. Roma-Bari: Laterza.

Cantone, Gaetana. 1992. Napoli barocca. Roma-Bari: Laterza.

Cantone, Gaetana (eds). 1992, Barocco Napoletano. Napoli: Istituto Poligrafico e Zecca dello Stato.

Chaussard, Jean Baptiste. 1801. Fêtes et Courtisanes de la Grèce, Supplément aux Voyages d'Anacharsis et d'Anteor. Paris.

Cicelyn, Edoardo. 2010. Piazza d'arte. Napoli 1995-2009. Quindici anni di installazioni in Piazza del Plebiscito. Napoli: Arte'm.

De Dominici, Bernado. 1745. Vite de' pittori, scultori ed architetti napoletani. Napoli.

De Seta, Cesare. 1991. Napoli fra Rinascimento e Illuminismo. Napoli: Electa.

Fagiolo, Marcello. 1967. Bernini. Una introduzione al gran teatro Barocco. Roma: Mario Bulzoni.

Doria, Gino. 1992. Piazza del Plebiscito. Napoli: Fausto Fiorentino Editore. 
Doria, Gino. 1986. I Palazzi di Napoli. Napoli: Guida.

Fagiolo, Marcello. 1983. Bernini e Roma: l'architettura e la citta. In AA.VV. Gian Lorenzo Bernini architetto e l'architettura europea del Sei-Settecento. Vol. II. Firenze: Istituto dell'Enciclopedia Italiana.

Fagiolo, Marcello (eds). 1997. La Festa a Roma dal Rinascimento al 1870. Torino: Umberto Allemandi editore.

Gadamer, Hans Georg. 1977. Die Actualitätdes Shönen, Kunstals Spiel Symbol und Fest. Stuttgart: Reclam.

Gambardella, Alfonso. 1968. Note su Ferdinando Sanfelice architetto napoletano. Napoli: Istituto Editoriale del Mezzogiorno.

Gambardella, Alfonso. 1974. Ferdinando Sanfelice. Napoli: Arti Grafiche Licenziato.

Gigli, Elena. 1997. Piazza di Spagna: apparati tra Francia e Spagna. In: Fagiolo dell'Arco, Maurizio (eds). La festa a Roma dal Rinascimento al 1870. Torino: Allemandi.

Gloton, Jean-Jacques. 1961. Les obélisque romains de la Renaissance au Néo-classicisme. In : Mélange d'Archéologie et de l'Ecole française de Rome. vol. 1, tome 73, p. 437-469.

Lattuada, Riccardo. 1997. La stagione del Barocco a Napoli. In AA.VV. Capolavori in festa. Effimero barocco a Largo di Palazzo (1683-1759). Napoli: Electa Napoli.

Lynch, Kevin. 1972. What Time is this Place? Cambridge: MIT Press.

Mancini, Franco. 1964. Scenografia napoletana dell'età barocca. Napoli: Edizioni Scientifiche Italiane.

Mancini, Franco. 1968. Feste ed apparati in Napoli dal Viceregno alla Capitale. Napoli: Edizioni Scientifiche Italiane.

Mancini, Franco. 1972. Feste, apparati e spettacoli teatrali in Storia di Napoli. Napoli: Edizioni Scientifiche Italiane.

Mancini, Franco; Gargano, Pietro. 1991. Piedrigrotta. Nel segno della tradizione, i luoghi, le feste e le canzoni. Napoli: Guida.

Monin, Eric. 2008. The speculative challenges of festival architecture in eighteenth century France. In: Bonnemaison, Sarah; Macy, Christine (eds). Festival Architecture. London: Routledge, p.155-180.

Oechslin, Werner; Buschow, Anja. 1987. Architecture de Fête, l'architecte metteur en scène. Bruxelles: Pierre Mardaga.

Pane, Roberto. 1939. Architettura dell'età Barocca in Napoli. Napoli: Editrice Politecnica.

Pane, Roberto (eds). 1984. Seicento napoletano. Arte, costume e ambiente. Milano: Edizioni di Comunità.

Salvatori, Gaia; Menzione, Corrado. 1984. Le guglie di Napoli: storia e restauro. Napoli: Electa Napoli. Zangheri, Luigi. 1996. Feste e apparati nella Toscana dei Lorena, 1737-1859. Firenze: L. S. Olschki.

\section{NOTES}

1. Traduction personnelle du texte en italien de W. Benjamin, Immagini di città (2007). 


\section{RÉSUMÉS}

L'article interroge l'origine historique du phénomène connu sous le nom d'«architecture éphémère » qui, ayant commencé à se développer à l'époque de la Rome baroque du Bernin, a modelé son expression caractéristique dans la culture et le langage qui ont fait la grandeur de la saison du baroque napolitain grâce à des personnalités telles que Cosimo Fanzago, Ferdinando Sanfelice, Domenico Antonio Vaccaro. Dans ce cadre, notre réflexion portant sur l'importance que la «machine de la fête baroque » a revêtu dans le développement urbain de la ville, la définition de l'espace collectif et la naissance de l'idée même de "place ", nous amènera à élargir l'horizon de notre étude sur le spectre de l'architecture, pour réfléchir à la force de renouveau et de persuasion que le "provisoire » est en mesure d'engendrer dans la vie des villes ainsi qu'au rôle que l'«éphémère " - en tant que catégorie pour ainsi dire anthropologique - a joué et continue de jouer dans le développement et dans la définition de l'espace urbain. L'analyse du cas d'étude représenté par l'espace urbain du Largo di Palazzo offrira quelques clés de lecture à propos de la déclinaison du concept d' "éphémère » dans le développement urbain, transposables à partir de l'expérience de la ville de Naples toute entière.

The contribution aims to investigate the historical origins of the phenomenon defined as "ephemeral architecture" that was first developed in the baroque Rome of Bernini and found its own expressive nature in the culture and language that made the Neapolitan baroque great, through the likes of Cosimo Fanzago, Ferdinando San Felice and Domenico Antonio Vaccaro. However, starting from a study of the significance that the macchina della festa barocca had on the urban development of the city, in defining the collective spaces and on the founding of the idea of the piazza, it will be possible to consider in a more general sense the power of renewal and persuasion that the provisional is capable of promoting in the life of cities over time, as well as on the role that the ephemeral, intended as an anthropological category, has had and continues to have on the development and definition of the concept of an urban space that is defined and measured through its rituals of use, its performances, the deep sense of wonder, amazement and participation that the ephemeral brings with it.

\section{INDEX}

Mots-clés : Éphémère, fête baroque, performance urbaine, Naples

Keywords : Ephemeral, Baroque, urban performance, Naples

\section{AUTEUR}

\section{ALESSANDRA CIRAFICI}

Alessandra Cirafici, Department of Architecture and Industrial Design, University of Campania Luigi Vanvitelli. She attained her degree in Architecture in 1989 from the Federico II University of Naples and received her PHD in "Rilievo e Rappresentazione del Costruito" in 1995. She is member of board PhD Program in "Architecture, Design and Cultural Heritage". She is dean of 
the Bachelor Degree in Fashion Design. Her field of research is the geometric-configurative representation of space, which investigates the problematic tied to the perceptual and communication processes in both architecture and the documentation of the extant as well as, more broadly, the project of visual communication.

alessandra.cirafici@unicampania.it 